\title{
High throughput resource efficient reconfigurable interleaver for MIMO WLAN application
}

\author{
Bijoy Kumar Upadhyaya ${ }^{1}$, Pijush Kanti Dutta Pramanik ${ }^{\text {Corresp., } 2 \text {, Salil Kumar Sanyal }}{ }^{3}$ \\ 1 Department of Electronics \& Communication Engineering, Tripura Institute of Technology, Narsingarh, Tripura, India \\ 2 Department of Computer Science \& Engineering, National Institute of Technology, Durgapur, West Bengal, India \\ 3 Department of Electronics \& Telecommunication Engineering, Jadavpur University, Kolkata, West Bengal, India \\ Corresponding Author: Pijush Kanti Dutta Pramanik \\ Email address: pkdp15ca1503@phd.nitdgp.ac.in
}

Demand for high-speed wireless broadband internet service is ever increasing. Multipleinput-multiple-output (MIMO) Wireless LAN (WLAN) is becoming a promising solution for such high-speed internet service requirements. This paper proposes a novel algorithm to efficiently model the address generation circuitry of the MIMO WLAN interleaver. The interleaver used in the MIMO WLAN transceiver has three permutation steps involving floor function whose hardware implementation is the most challenging task due to the absence of corresponding digital hardware. In this work, we propose an algorithm with a mathematical background for the address generator, eliminating the need for floor function. The algorithm is converted into digital hardware for implementation on the reconfigurable FPGA platform. Hardware structure for the complete interleaver, including the read address generator and memory module, is designed and modeled in VHDL using Xilinx Integrated Software Environment (ISE) utilizing embedded memory and DSP blocks of Spartan 6 FPGA. The functionality of the proposed algorithm is verified through exhaustive software simulation using ModelSim software. Hardware testing is carried out on Zynq 7000 FPGA using Virtual Input Output (VIO) and Integrated Logic Analyzer (ILA) core. Comparisons with few recent similar works, including the conventional Look-Up Table (LUT) based technique, show the superiority of our proposed design in terms of maximum improvement in operating frequency by $196.83 \%$, maximum reduction in power consumption by $74.27 \%$, and reduction of memory occupancy by $88.9 \%$. In the case of throughput, our design can deliver 8.35 times higher compared to IEEE 802.11n requirement. 


\section{High Throughput Resource Efficient Reconfigurable 2 Interleaver for MIMO WLAN Application}

3

4 5

6 7

- Narsingarh, Tripura, India

$8 \quad{ }^{2}$ Department of Computer Science \& Engineering, National Institute of Technology, Durgapur, 9 India

$10{ }^{3}$ Department of Electronics \& Telecommunication Engineering, Jadavpur University, Kolkata, 11 India

\section{Pijush Kanti Dutta Pramanik}

Department of Computer Science \& Engineering

National Institute of Technology, Durgapur, India

Email: pijushjld@yahoo.co.in, pkdp15ca1503@phd.nitdgp.ac.in 


\section{Abstract}

32

Demand for high-speed wireless broadband internet service is ever increasing. Multiple-inputmultiple-output (MIMO) Wireless LAN (WLAN) is becoming a promising solution for such high-speed internet service requirements. This paper proposes a novel algorithm to efficiently model the address generation circuitry of the MIMO WLAN interleaver. The interleaver used in the MIMO WLAN transceiver has three permutation steps involving floor function whose hardware implementation is the most challenging task due to the absence of corresponding digital hardware. In this work, we propose an algorithm with a mathematical background for the address generator, eliminating the need for floor function. The algorithm is converted into digital hardware for implementation on the reconfigurable FPGA platform. Hardware structure for the complete interleaver, including the read address generator and memory module, is designed and modeled in VHDL using Xilinx Integrated Software Environment (ISE) utilizing embedded memory and DSP blocks of Spartan 6 FPGA. The functionality of the proposed algorithm is verified through exhaustive software simulation using ModelSim software. Hardware testing is carried out on Zynq 7000 FPGA using Virtual Input Output (VIO) and Integrated Logic Analyzer (ILA) core. Comparisons with few recent similar works, including the conventional Look-Up Table (LUT) based technique, show the superiority of our proposed design in terms of maximum improvement in operating frequency by $196.83 \%$, maximum reduction in power consumption by $74.27 \%$, and reduction of memory occupancy by $88.9 \%$. In the case of throughput, our design can deliver 8.35 times higher compared to IEEE 802.11n requirement.

\section{Introduction}

The increasing use of multimedia services and the growth of graphics-based web contents have escalated the demand for high-speed wireless broadband communications. The use of more than one antenna at the transmitter and/or at the receiver aims to substantially improve the transmission/reception rate. Orthogonal Frequency Division Multiplexing (OFDM) is becoming a popular technique for high data rate wireless transmission [1]. OFDM may be combined with multiple antennas at both the access point and the mobile terminal to increase diversity gain and/or to enhance the system capacity on a time-varying multipath fading channel, resulting in a Multiple-Input-Multiple-Output (MIMO) OFDM system [2] [3]. 
60 The IEEE 802.11n, an amendment to the IEEE 802.11 standard, is based on MIMO-OFDM 61 transmission techniques to enable high-speed data communication with a maximum throughput 62 of $600 \mathrm{Mbps}$ [4]. In high-throughput wireless communication systems, interleavers [5] play an 63 important role in reducing the effect of a burst error in the channel and improve the performance 64 of Forward Error Correction (FEC) techniques.

65 66 67 68 69 70 71 72 73

In general terms, an interleaver consists of two parts: address generator and interleaver memory. The address generation process in MIMO WLAN transceiver is implementing the three steps in permutation wherein a floor function is involved [6]. But due to the non-availability of digital hardware in practice, it is difficult to implement the floor function. Due to this issue, the Look-up Table (LUT) based approach is generally used [7]. In a LUT-based approach, all possible addresses are pre-calculated and stored in the memory. The LUT-based technique is, in general, unattractive [8] as it requires a large number of memory blocks (LUTs) to house the addresses associated with different permissible modulation schemes, bandwidths (BWs), and spatial streams. In addition, the LUT-based address generator requires a large memory access time resulting in slower operation.

Literature review for MIMO WLAN transceiver implementation on hardware platform unveils some works. A work reported in [9] demonstrated the development of a prototype transceiver for IEEE 802.11a, followed by the upgradation to 1x4 MIMO WLAN. The authors claimed to implement the transceiver on Xilinx FPGA Virtex V LX330. Setiawan et al. [10] demonstrated prototyping of the 2X2 MIMO WLAN system using Register Transfer Level (RTL) design. The authors used the Model-Based Design Process (MBDP) for developing the RTL design of the transceiver and implemented on Altera FPGA Stratix-II EP2S180 and obtained maximum throughput of $144 \mathrm{Mbps}$. Another work for MIMO-OFDM transceiver implementation on Xilinx Virtex IV FPGA has been reported in [11]. Here, the authors implemented an interleaver and deinterleaver pair, including the convolutional channel coding algorithm of the MIMO-OFDM transceiver. This paper focuses on implementing the fundamental interleaving technique, which does not include inter-row, inter-column permutation, and frequency rotation parameters essential for the MIMO-OFDM transceiver. The FPGA implementation works presented in [9], [10], and [11] were neither aimed at full 4X4 MIMO-OFDM implementation nor achieving the $600 \mathrm{Mbps}$ throughput target. ASIC implementations of the MIMO-OFDM / IEEE 802.11n 
90 transceiver are described by some researchers in [12] [13]. Another recent work on designing an

91 FPGA-based address generator for a multi-standard interleaver is reported in the literature [14].

92 The authors made a combined implementation of the address generator for WLAN $(802.11 \mathrm{a} / \mathrm{b} / \mathrm{g})$,

93 WiMAX, and 3GPP LTE, but not of the MIMO WLAN (802.11n).

94 However, the implementations of [9], [10], [11], [12], [13], and [14] are not specifically focused 95 on interleaver/de-interleaver. They do not contain detailed implementation results leaving scope 96 for design optimization with respect to resource utilization, providing compact design, resulting 97 in higher throughput and reduced power consumption.

98 Very few papers reporting the hardware implementation of the MIMO WLAN interleaver are 99 available in the literature. In [15], Zhang et al. presented a de-interleaver address generator 100 implementation on a $0.13 \mu \mathrm{m}$ CMOS platform. The authors claimed that the implementation was 101 also done on the FPGA platform but without any implementation result. 2-D translation of the 102 interleaver equations for hardware simplicity was proposed in [7]. The final expressions, so derived, are very complex and do not clearly explain the hardware design issues, especially for 64-QAM. The implementation platform of this work is reported to be $65 \mathrm{~nm}$ CMOS technology. Another recent work [16] reported by the authors of [15] claimed betterment over their previous work in reducing complexity and improvement in maximum operating frequency keeping the same implementation platform. The improvement claimed by the authors is due to exchanging steps between the interleaver and the de-interleaver. In [17], the authors presented an FPGAbased implementation of the complete MIMO PHY modulator for IEEE 802.11n WLAN. The implementation is further extended to ASIC with $65 \mathrm{~nm}$ CMOS technology. The authors tabulated the FPGA and ASIC implementation results of the complete MIMO PHY modulator for IEEE 802.11n WLAN without mentioning the interleaver's resource occupancy or power consumption separately.

114 The above-mentioned issues have opened up further research scope for improving the implementation of the MIMO WLAN interleaver that complies with the high throughput data transmission requirements. In this paper, we propose a novel design of the interleaver used in a $4 \mathrm{x} 4$ MIMO WLAN transceiver. The proposed address generator algorithm eliminates the requirement of floor function from the address generator of the MIMO WLAN interleaver. 
119 The key contributions of this work are:

120 - The mathematical modeling of the new algorithm has been derived with general validity.

121

122

123

124

125

126

127

128

129

130

131

132

133

134

135

136

137

138

139

140

141

142

143

144

145

146

147

148
- The novel address generator algorithm has been generalized to accommodate more modulation schemes if required.

- The complete MIMO WLAN interleaver, including the proposed address generator algorithm, is transformed into digital hardware and implemented on Spartan 6 FPGA [18] using Xilinx ISE 12.1.

- To reduce the resource and power consumption and to enhance the throughput, the embedded resources of FPGA like dual-port Block RAM [19] and DSP blocks (DSP48A1) [20] have been successfully interfaced and utilized in the hardware model. This approach makes the design very compact and highly efficient. Comparison with existing similar works endorses the superiority of our proposed design in terms of multiple FPGA parameters.

- The functionality test of the address generator has been verified using ModelSim XE-III software.

- Further, hardware testing of the algorithm has also been carried out using Virtual Input and Output (VIO) and Integrated Logic Analyser (ILA) module on Zynq 7000 FPGA board, which further validates the proposed algorithm.

- The proposed design has been compared with a few recent implementations [7], [15], [16] by converting them into FPGA equivalent implementation using [21]. The comparison shows the superiority of the proposed design in terms of operating frequency, power consumption, and memory occupancy.

- The performance of the proposed interleaver is compared with IEEE 802.11n. The comparison results show that the proposed interleaver delivers much higher throughput than the maximum throughput requirement of IEEE 802.11n.

The rest of the paper is organized as follows. Section 2 presents the theoretical background of the interleaving process in the MIMO WLAN transceiver. Section 3 presents the proposed algorithm, including the mathematical background for the address generator. A description of the transformation of the proposed algorithm into digital hardware has been made in Section 4.

Simulation results followed by FPGA implementation details have been reported in Sections 5

PeerJ Comput. Sci. reviewing PDF | (CS-2020:10:54917:2:0:NEW 29 Apr 2021) 
149 and 6, respectively. The concluding remarks are given in Section 7.

\section{Interleaving in IEEE 802.11n}

151 In a MIMO WLAN transceiver, the encoded data stream obtained from the convolutional 152 encoder is fed to a special type of block interleaver. Interleaving in $802.11 \mathrm{n}$ is a three-step 153 process in which the first two steps provide spatial interleaving, and the final step performs

154 frequency interleaving [4]. The interleaving steps are defined in the form of three blocks shown 155 in Fig. 1. The first step $\left(B_{1}\right)$ ensures that adjacent coded bits are mapped onto non-adjacent 156 subcarriers, while the second step $\left(\mathrm{B}_{2}\right)$ is responsible for the mapping of adjacent coded bits 157 alternately onto less or more significant bits of the constellation, thus avoiding long runs of lowly 158 reliable bits. If more than one spatial stream exists in the $802.11 \mathrm{n}$ physical layer, the third step, 159 called frequency rotation $\left(\mathrm{B}_{3}\right)$, will be applied to the additional spatial streams. The frequency 160 rotation ensures that the consecutive carriers used across spatial streams are not highly 161 correlated.

Here, $\mathrm{N}$ is the block size corresponding to the number of coded bits per allocated sub-channels per OFDM symbol. $d$ represents the number of columns in the interleaver, whose values are 13 and 18 for $20 \mathrm{MHz}$ and $40 \mathrm{MHz}$ BW [5], respectively. The parameter $\mathrm{s}$ is defined as $s=\max (1$, $N_{B P S C S}$ ), whereas $N_{B P S C S}$ is the number of coded bits per sub-carrier and takes the values 1, 2, 4, and 6 for BPSK, QPSK, 16-QAM, and 64-QAM, respectively. $i_{s s}$ is the index of the spatial stream, and $N_{\text {rot }}$ is the parameter used for defining different rotations for the $20 \mathrm{MHz}$ and $40 \mathrm{MHz}$ cases. The operator $\%$ and \lfloor\rfloor represent modulo function and floor function, respectively.

\section{Proposed Algorithm for Address Generator of Interleaver}

171 The permutation steps described in the $\mathrm{B}_{1}, \mathrm{~B}_{2}$, and $\mathrm{B}_{3}$ blocks of Fig. 1 involve floor function. Our 172 objective is to propose a hardware-friendly algorithm to implement the address generator on a 173 reconfigurable platform that involves no floor function. Initially, a MATLAB program is 174 developed by implementing $B_{1}, B_{2}$, and $B_{3}$ blocks of Fig. 1 to determine the interleaver addresses 175 for all modulation schemes, spatial streams, and BWs. Table 1(a)-(c) show selected part these 
176 addresses for $N_{\text {bpscs }}=1, N=52, i_{s s}=4 ; N_{b p s c s}=4, N=208, i_{s s}=2$; and $N_{\text {bpscs }}=6, N=312, i_{s s}=3$

177 with $20 \mathrm{MHz}$ BW. Careful examination of these addresses reveals the correlation among them,

178 which may be proposed to express by new algorithms, as described in Table 2(a)-(c). The

179 complete mathematical formulations of the proposed algorithms, including all modulation

180 schemes, spatial streams, and BWs, are represented by Eq. 1-3.

181

Table 1(a) Part of interleaver write addresses with $N_{b p s c s}=1, N=52, i_{s s}=4, B W=20 M H z$

182

Table 1(b) Part of interleaver write addresses with $N_{\text {bpscs }}=4, N=208, i_{s S}=2, B W=20 \mathrm{MHz}$

184

185

186

Table 1(c) Part of interleaver write addresses with $N_{\text {bpscs }}=6, \mathrm{~N}=312, i_{s S}=3, \mathrm{BW}=20 \mathrm{MHz}$

$$
k_{n(Q P S K-B P S K)}= \begin{cases}D *(i+I)+(j+J) & \text { when } j<(D-J) \text { and } i<(C-I) \\ D *\{i-(C-I)\}+(j+J) & \text { when } j<(D-J) \text { and } i \geq(C-I) \\ D *(i+I+1)+\{j-(D-J)\} & \text { when } j \geq(D-J) \text { and } i<(C-I-1) \\ D *\{i-(C-I-1)\}+\{j-(D-J)\} & \text { when } j \geq(D-J) \text { and } i \geq(C-I-1)\end{cases}
$$

187

188

$$
k_{n(16-Q A M)}=
$$

$$
\begin{aligned}
& \left\{\begin{array}{l}
D *(i+I)+(j+J+1) \\
D *\{i-(C-I)\}+(j+J) \\
D *(i-(C-I))+(j+J+1) \\
D *(i+I)+(j+J-1) \\
D *(i-(C-I))+(j+J-1) \\
D *(i+I+1)+\{j-(D-J)\} \\
D *(i+I+1)+\{j-(D-J-1)\} \\
D *\{i-(C-I-1)\}+\{j-(D-J)\} \\
D *\{i-(C-I-1)\}+\{j-(D-J-1)\} \\
D *(i+I+1)+\{j-(D-J+1)\} \\
D *\{i-(C-I-1)\}+\{j-(D-J+1)\} \text { when }
\end{array}\right. \\
& \text { when }[(j<) \\
& \text { when }\{j<(D-J)\}[\{i<(C \text {. } \\
& \text { when }[\{j<(D-J)\} \&(j \% 2=0)] \&[\{i<(C-I)\} \&(i \% 2=1)] \\
& \text { when }\{j<(D-J)\} \&[\{i \geq(C-I)\} \&(i \% 2=0)] \\
& \text { when }[\{j<(D-J)\} \&(j \% 2=0)] \&[\{i \geq(C-I)\} \&(i \% 2=1)] \\
& \text { when }[\{j<(D-J)\} \&(j \% 2=1)] \&[\{i<(C-I)\} \&(i \% 2=1)] \\
& \text { when }[\{j<(D-J)\} \&(j \% 2=1)] \&[\{i \geq(C-I)\} \&(i \% 2=1)] \\
& \text { when }\{j \geq(D-J)\} \&[\{i<(C-I-1)\} \&(i \% 2=0)] \\
& \text { when }[\{j \geq(D-J)\} \&(j \% 2=0)] \&[\{i<(C-I-1)\} \&(i \% 2=1)] \\
& \text { when }\{j>=(D-J)\} \&[\{i \geq(C-I-1)\} \&(i \% 2=0)] \\
& \text { when }[\{j \geq(D-J)\} \&(j \% 2=0)] \&[\{i \geq(C-I-1)\} \&(i \% 2=1)] \\
& \text { when }[\{j \geq(D-J)\} \&(j \% 2=1)] \&[\{i<(C-I-1)\} \&(i \% 2=1)]
\end{aligned}
$$




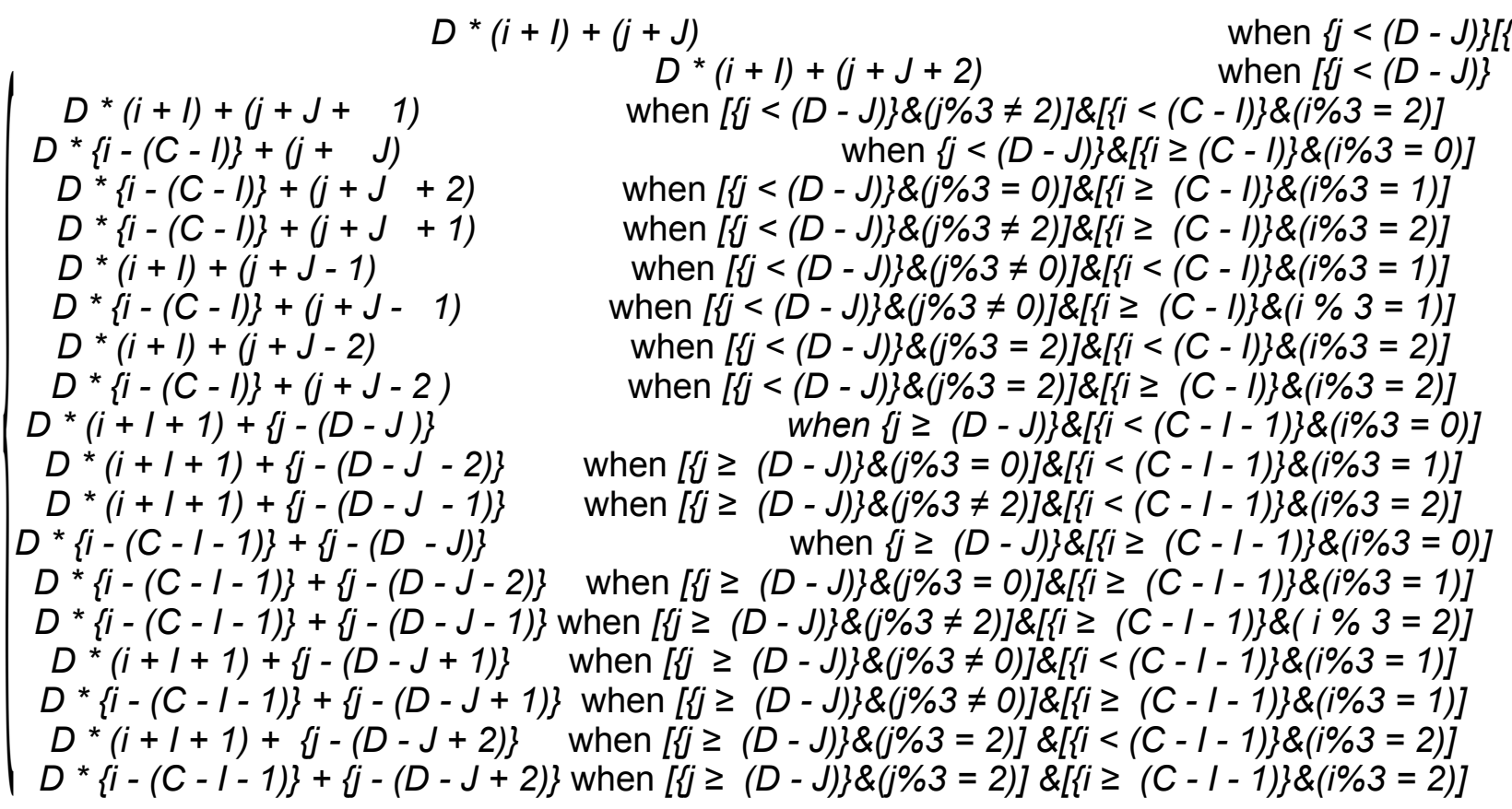

193

194

195

196

197

198

199

200

201

202

203

204

205

206

207

The general validity of the proposed mathematical formulation can be established with the help of [22]. As far as spatial permutation is concerned, the steps involved in IEEE 802.16e [22] and IEEE 802.11n [6] are identical. Additionally, the latter undergoes frequency rotation using the frequency interleaving step, as described by $\mathrm{B}_{3}$ in Fig. 1 for spatial streams other than the first. Further, analysis of the $3^{\text {rd }}$ step results that the entire term beyond $j_{k}$ (i.e., $J_{r o t}$ ) remains constant for a particular spatial stream and expressed by Eq. 4 [7].

$r_{k}=\left[j_{k}-J_{r o t}\right] \% N$

where $J_{\text {rot }}=\left[\left\{\left(i_{s S}-1\right) * 2\right\} \% 3+3\left[\frac{i_{s S}-1}{3}\right]\right] * N_{\text {rot }} * N_{B P S C S}$

As the first stream for all modulation schemes undergoes no frequency rotation, hence

$$
r_{k}=\left[j_{k}-0\right] \% N=\left[j_{k}\right] \% N=j_{k}
$$

For subsequent streams, the value of $J_{\text {rot }}$ differs for each spatial stream, modulation schemes, and BWs. All such possible values of $J_{r o t}$ are listed in Table 3. The expression of $j_{k}$ so derived for all modulation schemes in [22] if substituted in Eq. 1 gives three new equations. The final expressions obtained and the proposed mathematical formulations developed in this work 
208 generate the same results and are identical to results obtained through direct implementation of $209 \mathrm{~B}_{1}$ to $\mathrm{B}_{3}$ steps.

210

211

212

213

214

215

216

217

218

219

220

221

222

223

224

225

226

227

228

229

230

231

232

233

234

Table 2(a) Proposed algorithm for $N_{b p s c s}=1$ or 2 (BPSK / QPSK) with all $N, i_{s s}$, and BW

Table 2(b) Proposed algorithm for $N_{b p s c s}=4$ (16-QAM) with all $N, i_{s s}$, and $B W$

Table 2(c) Proposed algorithm for $N_{\text {bpscs }}=6(64-Q A M)$ with all $N, i_{s s}$, and $B W$

Table 3 Values of $J_{\text {rot }}$ for all modulation schemes, spatial streams, and BWs

The work reported in this paper includes interleaver design for all four modulation schemes (i.e., BPSK, QPSK, 16-QAM, and 64-QAM) as defined in the IEEE 802.11n standard. However, the proposed algorithm may be generalized, as follows, to include any other modulation scheme beyond the above standard.

1) Define the number of coded bits per sub-carrier $\left(N_{b p s c s}\right)$ for the modulation scheme beyond the above standard and compute $s=\max \left(1, N_{b p s c s}\right)$.

2) Define interleaver depth $(N)$, number of columns $(d)$, and compute the intermediate addresses after spatial interleaving $\left(j_{k}\right)$ by implementing $\mathrm{B}_{1}-\mathrm{B}_{2}$ steps.

3) Compute the final memory addresses $\left(r_{k}\right)$ by implementing step $\mathrm{B}_{3}$ with appropriate values of frequency rotation parameter $\left(N_{\text {rot }}\right)$ corresponding to the permissible bandwidths (BWs) for all four values of spatial streams $\left(i_{s s}\right)$.

4) Arrange the addresses obtained in step 3 in $\left(N / N_{r o t}\right) \times d$ tabular form with $j$ and $i$ as row and column numbers, respectively.

5) Identify the correlation between the subsequent addresses and re-arrange each address in $\left(N / N_{\text {rot }}\right) *\left(i \pm\right.$ offset $\left._{1}\right)+\left(j \pm o f f s e t_{2}\right)$ format. The offset $x_{x}=0$ with $i_{s s}=1$ for all values of $N$. All other values of offset $t_{x}$ to be computed using the correlation between the subsequent addresses. 
235

236

237

238

239

240

241

242

243

244

245

246

247

248

249

250

251

252

253

254

255

256

257

258

259

260

261

6) Group the above mathematical expressions (obtained from step 5) according to the specific modulation scheme. These expressions exclude floor function, hence, suitable for implementation on the hardware platform.

7) Functional verification of the algorithm may be carried out by comparing the addresses of steps 3 and 6 using suitable software.

\section{Transformation into Hardware}

This section describes the transformation of the proposed address generator algorithm into digital hardware. The top-level view of the complete interleaver consisting of the proposed address generator and memory block is shown in Fig. 2.

\subsection{Memory Block}

The detailed arrangement of the memory block for one spatial stream having a similar structure as in [23] is shown in Fig. 3. The structure is generic and applies to all spatial streams. It receives three inputs from the address generator block; write address $\left(W A_{x}\right)$, read address $\left(R A_{x}\right)$, and $\operatorname{sel}_{x}$. The requirement of two memory blocks for block interleaving is accomplished here with a dualport memory (with Port A and B) where the read and write operations can be performed simultaneously. As seen in Fig. 3, the first $288 \mathrm{H}$ locations are used as Port A, and the next $288 \mathrm{H}$ locations as Port B. An adder is used to insert the bias of $288 \mathrm{H}$ while generating the addresses for Port B. When one port is being written, another one is read, and vice versa. Swapping between read/write operations at the end of a cycle is performed using the signal sel $_{x}$, generated using a toggle flip flop.

\section{Fig. 2. A top-level view of the complete interleaver \\ Fig. 2. A top-level view of the complete interleaver}

\subsection{Address Generator}

The address generator is the heart of the interleaver. The encoding schemes used in this work for the two inputs, $B W$ and $N_{c b p s c}$ of the address generator, are described in Table 4 . The $i_{s s}-i_{s s 4}$ represent the four different spatial streams of the address generator, each consisting of write 
$262\left(W A_{x}\right)$, read $\left(R A_{x}\right)$ address, and select signal ( $\left.s e l_{x}\right)$ output. As shown in Fig. 4, in the write address 263 generator, a multiplexer is used to route the desired $W A_{x}$ from four possible sources based on the 264 value of $N_{c b p s c}$ for a particular spatial stream, $I_{s s x}$.

265 Figure 5(a) and (b) show the hardware used for the generation of row-count (JCOUNT) and 266 column-count (ICOUNT), respectively, which consist of up-counters and comparators. As per 267 [6], the column number is defined as $\mathrm{C}=13$ and 18 , for $\mathrm{BW}=20 \mathrm{MHz}$ and $40 \mathrm{MHz}$, 268 respectively. Circuit arrangement for the generation of row number, $D$ using $B W$ and $N_{c b p s c}$ is 269 shown in Fig. 6. Similarly, Fig. 7(a) and (b) describe hardware used for the generation of $270 I C O U N T<\left(C-I_{x}\right), I C O U N T \geq\left(C-I_{x}\right)$, JCOUNT $<\left(D-J_{x}\right)$, and $J C O U N T \geq\left(D-J_{x}\right)$ signals. Here $I_{x}$ 271 and $J_{y}$ are the column and row offset values, respectively, which are used while computing the 272 addresses, and are defined in Table 5.

273

274

Table 4(a) Encoding of $B W$

275

276

Table 4(b) Encoding of $N_{c b p s c}$

277

278

Fig. 3. Internal structure of memory block

279

280

281

282

Fig. 5. Scheme showing the generation of (a) row-count and (b) column-count

283

284

The hardware required for the generation of $R A_{x}$ is shown in Fig. 8. Like the write address 285 generator, the structure developed for the generation of $R A_{x}$ is also generic and is applicable to 286 all the spatial streams. The first and second level multiplexers select one of the values of 287 interleaver depth from the inputs with $B W$ and mod_typ signal. The $r d$ _count is a 10-bit up counter and generates $R A_{x}$. While progressing through the count values, when the $r d$ count value 289 equals the output of $\mathrm{M}_{1}$, a reset pulse is generated by the comparator, and rd_count goes to the 290 initial state to start another cycle. 
293

294

295

296

297

298

299

300

301

302

303

304

305

306

307

308

309

310

311

312

313

314

315

316

317

318

319

320

321

322
Fig. 7 Arrangement showing the generation of (a) ICOUNT $<\left(C-I_{x}\right)$ and ICOUNT $\geq\left(C-I_{x}\right)$

(b) $\operatorname{JCOUNT}<\left(D-J_{y}\right)$ and $J C O U N T \geq\left(D-J_{y}\right)$

Table 5 Definition of $I_{x}$ and $J_{y}$ for all streams and $B W$

Figures 9 and 10 show the rest of the circuit details required to generate $W A_{x}$ with BPSK/QPSK, 16-QAM, and 64-QAM modulation schemes. In these figures, the adders $\left(\mathrm{A}_{1}, \mathrm{~A}_{2}\right.$, and $\left.\mathrm{A}_{3}\right)$ receive two inputs; one from the row-count part (purple colored) and the other from the columncount part (blue colored) of the circuit. In Fig. 9, the JCOUNT $+J_{y}$ signal is generated by an adder $\left(\mathrm{A}_{4}\right)$, whereas the two subtractors $\left(\mathrm{S}_{1}\right.$ and $\left.\mathrm{S}_{2}\right)$ generate the signal JCOUNT - $\left(D-J_{y}\right)$. Based on the value of $J C O U N T<\left(D-J_{y}\right)$ signal, the multiplexer $\left(\mathrm{M}_{2}\right)$ routes one of these signals to the input of the $\mathrm{A}_{1}$. Similar hardware structures can be found to generate signals like ICOUNT $+I_{x}$, $I C O U N T+I_{x}+1$, ICOUNT $-\left(C-I_{x}\right)$, etc., in the column-count part. The column-count part's output gets multiplied with $D$ in the multiplier $\left(\mathrm{ML}_{1}\right)$ to generate the second input of $\mathrm{A}_{1}$. In Fig. 10 , the circuit details for generating signals like ICOUNT $+I_{x}, I C O U N T-\left(C-I_{x}\right), J C O U N T+$ $J_{y}, J C O U N T-\left(D-J_{y}\right)$, etc., are not shown to avoid repetition and clumsiness. The condition for the generation of select inputs $(I I 4, J J 4, I I 6$, and JJ6) for the multiplexers of Fig. 10, are described and encoded in Table 6(a) and (b).

Fig. 8. Circuit for the generation of read address $\left(R A_{x}\right)$

Fig. 9. Circuit diagram for the generation of interleaver write addresses with $N_{\text {cbpsc }}=1$ or 2

Fig. 10. Circuit diagram for the generation of interleaver write addresses with (a) $N_{c b p s c}=4$ and (b) $N_{\text {cbpsc }}=6$

Table 6(a) Encryption of signals II4 and JJ4

Table 6(b) Encryption of signals II6 and JJ6 
323

324

325

326

327

328

329

330

331

332

333

334

335

336

337

338

339

340

341

342

343

344

345

346

347

348

349

350

351

\section{Simulation Results}

The digital hardware of the MIMO WLAN interleaver is translated into a VHDL program using Xilinx ISE 12.1. The proposed design of the interleaver is simulated, and the functionality verification is done using ModelSim XE-III. The address generation circuitry of the interleaver is tested for all BWs, spatial streams, and modulation schemes, out of which two results (for $B W=$ $0, N_{b p s c s}=00$ and $B W=1, N_{b p s c s}=11$ ) are presented in Fig. 11(a)-(b). The last four signals (int_add_1 to int_add_4) of Fig. 11(a) and (b) show the sequence of write addresses generated in synchronization with a clock signal (clk) for all the four spatial streams of the interleaver ( $I_{s s}$ -

$\left.I_{s S}\right)$. The write address sequence generated by the proposed interleaver for spatial stream 1 (i.e., int_add_1) is $0,4,8,12, \ldots$ Similarly, the address sequence for spatial stream 2 (i.e., int_add_2) is $26,30,34$, and so on. The last address sequence (i.e., int_add_4) of Fig. 11(a) tallies with the address sequences shown in Table 1(a). Automatic address verification has also been carried out between the addresses generated by our proposed algorithm and the addresses obtained through steps $B_{1}-B_{3}$ of Section 2 involving floor function by running a separate MATLAB program. This verification further endorses the correctness of the proposed algorithm.

Fig. 11. Write addresses (WAx) for (a) $B W=0$ (20MHz), $N_{b p s c s}=00$ (BPSK), (b) $B W=1(40 M H z)$,

$$
N_{\text {bpscs }}=11 \text { (64-QAM) }
$$

\section{FPGA Implementation Results}

The proposed design of the interleaver is transformed into a VHDL model using Xilinx ISE 12.1 and is implemented on Xilinx Spartan-6 FPGA. Despite our exhaustive literature survey, any similar implementation on the FPGA platform has not been noticed. As a result, the conventional LUT-based approach has been implemented on the same FPGA platform utilizing Block RAM (BRAM) to house the address LUTs. Four dual port BRAM memory blocks are used to implement the interleaver memory in both designs. Comparative analysis of the two implementations in terms of device utilization is made in Table 7. The betterment of the proposed technique can be quantified in terms of embedded memory utilization (88.9\% memory block saving) and operating speed (37.8\% speed improvement). The use of DSP blocks as multiplier improves the performance of the circuit by reducing delay. The circuit works at a 
352 maximum clock frequency $(f)$ of $208.7 \mathrm{MHz}$ with $28.62 \mathrm{~mW}$ of total power consumption, which 353 includes static and dynamic power. The use of FPGA's embedded DSP blocks (DSP48A1s) as a 354 multiplier and embedded dual-port memory (BRAM) helps to reduce the memory access time 355 and, in turn, improves the throughput of the system. Resource efficiency and compact design are 356 the key contributors to reducing the power consumption of the interleaver. In addition, Spartan 6 357 FPGA itself is known for better power efficiency, increased productivity, and higher 358 performance implementation platform.

359

360

361

362 363 364 365 366 367 368

369

370

371

372

373

374 375 376 377 378 379 380 381 382

77

\section{Table 7 Device Utilization Summary}

The hardware testing of the address generator for the MIMO WLAN interleaver has been performed using VIO and ILA. VIO and ILA are the customizable cores that facilitate both monitoring and driving internal FPGA signals in real-time. Fig. 12 shows the block level design of the test environment using VIO and ILA wherein the proposed address generator block (WLAN_MIMO_NEW_0) is placed in the middle of the VIO (left side) and ILA (right side) blocks. The VIO injects user-defined RESET, BW, and NBPSCS signals. The outputs generated by the address generator (INT_ADD_1, 2, 3, and 4) are fed to the ILA and VIO for verification. An external clock (clk) signal drives all the modules synchronously.

(1)

Fig. 12. Test arrangement of the address generator using VIO and ILA

The throughputs of the proposed interleaver for all four modulation schemes are computed using Eq. 5 and presented in Table 8.

$$
T p=f \times N_{b p s c s} \times i_{s s}
$$

\section{Table 8 Throughput comparison with IEEE 802.11n}

The last column of Table 8 justifies our high throughput claim of the proposed interleaver. This provides the opportunity to implement the proposed design in relatively slower and lower-cost FPGAs as well, thereby providing a cost-effective solution. 
383 Besides, a comparison with few works has been made based on the equivalence drawn between 384 FPGA and ASIC implementations in [21]. The comparative study of the proposed 385 implementation regarding key FPGA parameters shows betterment over other similar recent 386 works and is presented in Table 9 and Fig. 13. The proposed circuit shows betterment over [7], 387 [15], [16], and LUT-based technique in terms of maximum operating frequency. In terms of 388 power consumption, our implementation is found to be the most efficient among the designs 389 presented in [7], [15], and [16] of Fig. 13. As direct implementation of floor function is not possible, improvement in terms of memory block used (BRAM) and clock frequency over the LUT-based technique may be considered as the performance improvement of our novel 392 algorithm due to the elimination of floor function from the interleaver address generator circuitry.

Fig. 13 Performance comparison with [15], [7], [16], and LUT-based work

Massive MIMO system, a key technology being deployed in the 5G system, employs an array of a large number of transmitting antennas at the base station to achieve high throughput has been investigated to compare our FPGA implementation results. Tan et al. [24] have demonstrated CMOS implementation of the message-passing detector (MPD) designed for a 256-QAM massive MIMO system supporting 32 concurrent mobile users in each time-frequency resource with $2.76 \mathrm{Gbps}$ throughput. As far as throughput is concerned, our proposed interleaver on the FPGA platform shows a competitive result with that of [24].

\section{Conclusions}

407 This work demonstrates the design and implementation of novel interleaver hardware on the FPGA platform to be used in OFDM-based MIMO WLAN applications. A new algorithm has been proposed for the address generator of the interleaver eliminating the requirement of floor

410 function, and is supported by the mathematical formulation with general validity. The algorithm

411 is transformed into the digital circuit and is modeled using VHDL software. Simulation results 
412 and hardware testing verify the functionality of the proposed algorithm. Hardware

413 implementation of the VHDL model using Xilinx ISE is done and is tested on Xilinx Spartan 6

414 FPGA. Efficient design and use of FPGA's embedded resources during implementation enables

415 betterment over a few recent similar works and conventional design in terms of multiple FPGA

416 parameters and the interleaver throughput.

\section{References}

418 [1] Yang H. 'A road to future broadband wireless access: MIMO-OFDM-based air

[2] Bolcskei H., 'MIMO-OFDM wireless systems: basics, perspectives and challenges,' IEEE Wireless Communication, 2006, vol. 13 (4), pp. 31-37.

[3] Hwang T., Yang C., Wu G., et al. 'OFDM and its wireless applications: a survey', IEEE Transaction on Vehicular Technology, 2009, vol. 58 (4), pp. 1673-1694.

[4] Paul T. K. \& Ogunfunmi T., 'Wireless LAN comes of age: understanding the IEEE 802.11n amendment' IEEE Circuit and System Magazine, 2008, vol. 8 (1), pp. 28-54.

[5] Niu H., Ouyang X., Ngo C., 'Interleaver design for MIMO OFDM based wireless LAN', Proc. IEEE Wireless Communication and Networking Conference, vol. 4, Las Vegas, USA, 2006, pp. 1825-1829.

[6] IEEE 802.11n-2009: 'Wireless LAN Medium Access Control (MAC) and Physical Layer (PHY) specifications: enhancements for higher throughput,' The IEEE Standards Association, New York, NY, USA, 2009.

[7] Asghar R. and Liu D., 'Low complexity hardware interleaver for MIMO-OFDM based wireless LAN,' Proc. Int. IEEE Symp. on Circuits and Systems (ISCAS), Taipai, 2009, pp.1747-1750.

[8] Upadhyaya B. K., and Sanyal S. K., 'Efficient FPGA implementation of address generator for WiMAX de-interleaver,' IEEE Transaction on Circuits and Systems - II : Express Briefs, 2013, vol. 60(8), pp. 492-496.

[9] Eickhoff R., Tittelbach-Helmrich K., Wickert M., et al. 'Physical layer amendments for MIMO features in 802.11a', Proc. of Conf. Future Network \& Mobile Summit, 2011, pp.18.

[10] Setiawan H., Nagao Y., Kurosaki M., et al. 'IEEE 802.11n Physical Layer Implementation on Field Programmable Gate Array', TELKOMNIKA Indonesian Journal of Electrical Engineering, 2011, vol. 10 (1), pp.67-74.

[11] Srinandhini K., and Vaithianathan V., "FPGA Implementation of MIMO-OFDM Transceiver" International Conference on Communication and Signal Processing, India, 2014, pp. 353-357.

[12] Perels D., Haene S., Luethi P., et al. 'ASIC implementation of a MIMO-OFDM transceiver for 192 mbps WLANs' Proc. of ESSCIRC, France, 2005, pp. 215-218. 
449

450

451

452

453

454

455

456

457

458

459

460

461

462

463

464

465

466

467

468

469

470

471

472

473

474

475

476

477

478

479
[13] Tran T.H., Nagao Y., Kurosaki M, et al. 'ASIC implement of 600 Mbps IEEE 802.11n $4 \times 4$ MIMO wireless LAN system', 14th IEEE Int. Conf. on Advan. Commu. Tech. (ICACT) Pyeongchang, Korea, 2012, pp. 360-363.

[14] Babu G. S., Gopalakrishnan L., "Reconfigurable address generator for multi-standard interleaver", Microprocessors and Microsystems, 2019, vol. 65, pp. 47-56.

[15] Zhang Z.-D., Wu B., Zhu Y.-X., et al. 'Design and implementation of a multi-mode interleaver/deinterleaver for MIMO OFDM systems,' Proc. of the 8th IEEE Int. Conf. on ASIC (ASICON' 09), Changsha, China, 2009, pp.513-516.

[16] Zhang Z., Wu B., Zhou Y. et al. 'Low-Complexity Hardware Interleaver / Deinterleaver for IEEE 802.11a/g/n WLAN' VLSI Design, Hindwai Publisher, 2012, pp. 1-7.

[17] Kim M., Kim J., "An Implementation of MIMO PHY Modulator for IEEE 802.11n WLAN System", IDEC Journal of Integrated Circuits and Systems, 2017, vol. 3, (1).

[18] Xilinx, 'Spartan-6 Data sheet', 30 January 2015. [Online]. Available: https://www.xilinx.com/support/documentation/data_sheets/ds162.pdf. [Accessed 10 January 2018].

[19] Xilinx, 'Spartan-6 FPGA Block RAM resources user guide', 8 July 2011. [Online]. Available: https://www.xilinx.com/support/documentation/user_guides/ug383.pdf. [Accessed 12 January 2018].

[20] Xilinx, 'Spartan-6 FPGA DSP48A1 Slice user guide', 24 June 2009. [Online]. Available: https://www.xilinx.com/support/documentation/user_guides/ug389.pdf. [Accessed 12 January 2018].

[21] Kuon I. and Rose J., 'Measuring the gap between FPGAs and ASICs,' Proc. Int. Symposium on Field Programmable Gate Arrays, California, USA, 2006, pp. 21-30.

[22] Asghar R. and Liu D., '2D realization of WiMAX channel interleaver for efficient hardware implementation,' Proc. of World Academic Science Engineering Technololgy, Hong Kong, 2009, vol. 51, pp. 25-29.

[23] Upadhyaya B. K., and Sanyal S. K., "An improved LUT based reconfigurable multimode interleaver for WLAN application," Int. J. of Recent Trends in Engg. and Tech., ACEEE, 2011, vol. 6 (2), pp.183-188.

[24] Tang W., Chen C. H., and Zhang Z., "A 0.58mm2 2.76Gb/s 79.8pJ/b 256-QAM massive MIMO message-passing detector," Proc. in IEEE Symp. VLSI Circuits, Jun. 2016, pp. 1-2. 


\section{Table $\mathbf{1}$ (on next page)}

Part of interleaver write addresses

Table 1(a) Part of interleaver write addresses with $N_{\text {bpscs }}=1, N=52, i_{s s}=4, B W=20 \mathrm{MHz}$

Table 1(b) Part of interleaver write addresses with $N_{\text {bpscs }}=4, N=208, i_{s s}=2, B W=20 M H z$

Table 1(c) Part of interleaver write addresses with $N_{\text {bpscs }}=6, N=312, i_{s s}=3, B W=20 \mathrm{MHz}$ 
1 2

4
5
6
7

8

10

11

Table 1(a) Part of interleaver write addresses with $N_{b p s c s}=1, N=52, i_{s s}=4, B W=20 \mathrm{MHz}$

\begin{tabular}{ccccccccc}
\hline & \multicolumn{10}{c}{ Column no(i) } \\
\hline Row no(j) & 0 & 1 & 2 & $\ldots$ & 9 & 10 & 11 & 12 \\
\hline $\mathbf{0}$ & 13 & 17 & 21 & $\ldots$ & 49 & 1 & 5 & 9 \\
$\mathbf{1}$ & 14 & 18 & 22 & $\ldots$ & 50 & 2 & 6 & 10 \\
$\mathbf{2}$ & 15 & 19 & 27 & $\ldots$ & 51 & 3 & 7 & 11 \\
$\mathbf{3}$ & 16 & 20 & 28 & $\ldots$ & 0 & 4 & 8 & 12 \\
\hline
\end{tabular}

Table 1(b) Part of interleaver write addresses with $N_{b p s c s}=4, N=208, i_{s s}=2, B W=20 \mathrm{MHz}$

\begin{tabular}{cccccccccc}
\hline & \multicolumn{7}{c}{ Column no(i) } \\
\hline Row no(j) & 0 & 1 & 2 & $\ldots$ & 6 & 7 & 8 & $\ldots$ & 12 \\
\hline $\mathbf{0}$ & 104 & 121 & 136 & $\ldots$ & 200 & 9 & 24 & $\ldots$ & 88 \\
$\mathbf{1}$ & 105 & 120 & 137 & $\ldots$ & 201 & 8 & 25 & $\ldots$ & 89 \\
$\mathbf{2}$ & 106 & 123 & 138 & $\ldots$ & 202 & 11 & 26 & $\ldots$ & 90 \\
$\ldots$ & $\ldots$ & $\ldots$ & $\ldots$ & $\ldots$ & $\ldots$ & $\ldots$ & & $\ldots$ & $\ldots$ \\
$\mathbf{7}$ & 111 & 126 & 143 & $\ldots$ & 207 & 14 & 31 & $\ldots$ & 95 \\
$\mathbf{8}$ & 112 & 129 & 144 & $\ldots$ & 0 & 17 & 32 & $\ldots$ & 96 \\
$\mathbf{9}$ & 113 & 128 & 145 & $\ldots$ & 1 & 16 & 33 & $\ldots$ & 97 \\
$\mathbf{1 0}$ & 114 & 131 & 146 & $\ldots$ & 2 & 19 & 34 & $\ldots$ & 98 \\
$\ldots$ & $\ldots$ & $\ldots$ & $\ldots$ & $\ldots$ & $\ldots$ & $\ldots$ & & $\ldots$ & $\ldots$ \\
$\mathbf{1 5}$ & 119 & 134 & 151 & $\ldots$ & 7 & 22 & 39 & $\ldots$ & 103 \\
\hline
\end{tabular}

Table 1(c) Part of interleaver write addresses with $N_{b p s c s}=6, N=312, i_{s s}=3, B W=20 \mathrm{MHz}$

\begin{tabular}{cccccccccc}
\hline & \multicolumn{7}{c}{ Column no(i) } \\
\hline Row no(j) & 0 & 1 & 2 & 3 & 4 & 5 & 6 & $\ldots$ & 12 \\
\hline $\mathbf{0}$ & 234 & 260 & 283 & 306 & 20 & 43 & 66 & $\ldots$ & 210 \\
$\mathbf{1}$ & 235 & 258 & 284 & 307 & 18 & 44 & 67 & $\ldots$ & 211 \\
$\mathbf{2}$ & 236 & 259 & 282 & 308 & 19 & 42 & 68 & $\ldots$ & 212 \\
$\ldots$ & $\ldots$ & $\ldots$ & $\ldots$ & $\ldots$ & $\ldots$ & $\ldots$ & & $\ldots$ & $\ldots$ \\
$\mathbf{5}$ & 239 & 262 & 285 & 311 & 22 & 45 & 71 & $\ldots$ & 215 \\
$\mathbf{6}$ & 240 & 266 & 289 & 0 & 26 & 49 & 72 & $\ldots$ & 216 \\
$\mathbf{7}$ & 241 & 264 & 290 & 1 & 24 & 50 & 73 & $\ldots$ & 217 \\
$\mathbf{8}$ & 242 & 265 & 288 & 2 & 25 & 48 & 74 & $\ldots$ & 218 \\
$\ldots$ & $\ldots$ & $\ldots$ & $\ldots$ & $\ldots$ & $\ldots$ & $\ldots$ & $\ldots$ & $\ldots$ & $\ldots$ \\
$\mathbf{2 3}$ & 257 & 280 & 303 & 17 & 40 & 63 & 89 & $\ldots$ & 233 \\
\hline
\end{tabular}

12 


\section{Table 2 (on next page)}

Proposed algorithm

Table 2(a) Proposed algorithm for $N_{\text {bpscs }}=1$ or 2 (BPSK / QPSK) with all $N, i_{s s}$ and BW Table 2(b) Proposed algorithm for $N_{b p s c s}=4$ (16-QAM) with all $N, i_{s s}$ and BW Table 2(c) Proposed algorithm for $N_{\text {bpscs }}=6(64-Q A M)$ with all $N, i_{s s}$ and $B W$ 
1 2 3

Table 2(a) Proposed algorithm for $N_{b p s c s}=1$ or 2 (BPSK / QPSK) with all $N, i_{s s}$ and $B W$

\begin{tabular}{|c|c|c|c|c|c|c|c|c|c|c|}
\hline \multicolumn{2}{|c|}{ Column no. $(i) \rightarrow$} & 0 & 1 & 2 & 3 & $\cdots$ & $C-4$ & $C-3$ & $C-2$ & $C-1$ \\
\hline \multicolumn{2}{|c|}{$V^{\text {Row no. }(j)}$} & \multicolumn{4}{|c|}{$i<(C-I)$} & & \multicolumn{4}{|c|}{$i>=(C-I)$} \\
\hline 0 & \multirow{4}{*}{$j<(D-J)$} & \multirow{4}{*}{\multicolumn{4}{|c|}{$D *(i+I)+(j+J)$}} & \multirow{4}{*}{$\cdots$} & \multirow{4}{*}{\multicolumn{4}{|c|}{$D *\{i-(C-I)\}+(j+J)$}} \\
\hline 1 & & & & & & & & & & \\
\hline 2 & & & & & & & & & & \\
\hline 3 & & & & & & & & & & \\
\hline \multicolumn{2}{|r|}{$\cdots$} & \multicolumn{4}{|c|}{$i<(C-I-1)$} & & \multicolumn{4}{|c|}{$i>=(C-I-1)$} \\
\hline$D-4$ & \multirow{4}{*}{$j>=(D-J)$} & \multirow{4}{*}{\multicolumn{4}{|c|}{$D *(i+I+1)+\{j-(D-J)\}$}} & \multirow{4}{*}{$\ldots$} & \multirow{4}{*}{\multicolumn{4}{|c|}{$D^{*}\{i-(C-I-1)\}+\{j-(D-J)\}$}} \\
\hline$D-3$ & & & & & & & & & & \\
\hline$D-2$ & & & & & & & & & & \\
\hline$D-1$ & & & & & & & & & & \\
\hline
\end{tabular}

Table 2(b) Proposed algorithm for $N_{b p s c s}=4$ (16-QAM) with all $N, i_{s s}$ and $B W$

\begin{tabular}{|c|c|c|c|c|c|c|c|c|c|c|}
\hline \multicolumn{2}{|c|}{ Column no. $(i) \rightarrow$} & 0 & 1 & 2 & 3 & $\cdots$ & $C-4$ & $C-3$ & $C-2$ & $C-1$ \\
\hline \multicolumn{2}{|c|}{$\downarrow \quad$ Row no. $(j)$} & \multicolumn{2}{|c|}{$\begin{array}{c}\{i<(C-I)\} \\
\&(i \% 2=0)\end{array}$} & \multicolumn{2}{|c|}{$\begin{array}{c}\{i<(C-I)\} \\
\&(i \% 2=1)\end{array}$} & & \multicolumn{2}{|c|}{$\begin{array}{c}i>=(C-I) \\
\&(i \% 2=0)\end{array}$} & \multicolumn{2}{|c|}{$\begin{array}{c}i>=(C-I) \\
\&(i \% 2=1)\end{array}$} \\
\hline$\frac{0}{1}$ & $\begin{array}{c}\{j<(D-J)\} \\
\&(j \% 2=0)\end{array}$ & \multicolumn{2}{|c|}{$\begin{array}{c}D *(i+I)+ \\
(j+J) \\
\end{array}$} & \multicolumn{2}{|c|}{$\begin{array}{c}D^{*}(i+I)+ \\
(j+J+1)\end{array}$} & \multirow[b]{2}{*}{$\cdots$} & \multicolumn{2}{|c|}{$\begin{array}{c}D^{*}\{i-(C-I)\}+ \\
(j+J)\end{array}$} & \multicolumn{2}{|c|}{$\begin{array}{c}D^{*}(i-(C-I))+ \\
(j+J+1)\end{array}$} \\
\hline$\frac{2}{3}$ & $\begin{array}{l}\{j<(D-J)\} \\
\&(j \% 2=1)\end{array}$ & \multicolumn{2}{|c|}{$\begin{array}{c}D^{*}(i+I)+ \\
\quad(j+J)\end{array}$} & \multicolumn{2}{|c|}{$\begin{array}{c}D^{*}(i+I)+ \\
(j+J-1)\end{array}$} & & \multicolumn{2}{|c|}{$\begin{array}{c}D *(i-(C-I))+ \\
(j+J)\end{array}$} & \multicolumn{2}{|c|}{$\begin{array}{c}D^{*}(i-(C-I))+ \\
\quad(j+J-1)\end{array}$} \\
\hline \multicolumn{2}{|r|}{$\cdots$} & \multicolumn{2}{|c|}{$\begin{array}{c}i<(C-I-1) \\
\&(i \% 2=0)\end{array}$} & \multicolumn{2}{|c|}{$\begin{array}{l}\{i<(C-I-1)\} \\
\&(i \% 2=1)\end{array}$} & & \multicolumn{2}{|c|}{$\begin{array}{l}i>=(C-I-1) \\
\&(i \% 2=0)\end{array}$} & \multicolumn{2}{|c|}{$\begin{array}{l}i>=(C-I-1) \\
\&(i \% 2=1)\end{array}$} \\
\hline$\frac{D-4}{D-3}$ & $\begin{array}{l}\{j>=(D-J)\} \\
\&(j \% 2=0)\end{array}$ & \multicolumn{2}{|c|}{$\begin{array}{c}D *(i+I+1)+ \\
\{j-(D-J)\}\end{array}$} & \multicolumn{2}{|c|}{$\begin{array}{c}D *(i+I+1)+ \\
\{j-(D-J-1)\}\end{array}$} & & \multicolumn{2}{|c|}{$\begin{array}{c}D^{*}\{i-(C-I-1)\}+ \\
\{j-(D-J)\}\end{array}$} & \multicolumn{2}{|c|}{$\begin{array}{c}D *\{i-(C-I-1)\}+ \\
\quad\{j-(D-J-1)\}\end{array}$} \\
\hline$D-2$ & \multirow{2}{*}{$\begin{array}{l}\{j>=(D-J)\} \\
\&(j \% 2=1)\end{array}$} & \multirow{2}{*}{\multicolumn{2}{|c|}{$\begin{array}{c}D *(i+I+1)+ \\
\quad(j-(D-J))\end{array}$}} & \multirow{2}{*}{\multicolumn{2}{|c|}{$\begin{array}{l}D^{*}(i+I+1)+ \\
\{j-(D-J+1)\}\end{array}$}} & & \multirow{2}{*}{\multicolumn{2}{|c|}{$\begin{array}{c}D^{*}\{i-(C-I-1)\}+ \\
\{j-(D-J)\}\end{array}$}} & \multirow{2}{*}{\multicolumn{2}{|c|}{$\begin{array}{c}D *\{i-(C-I-1)\}+ \\
\{j-(D-J+1)\}\end{array}$}} \\
\hline$D-1$ & & & & & & & & & & \\
\hline
\end{tabular}

Table 2(c) Proposed algorithm for $N_{b p s c s}=6$ (64-QAM) with all $N, i_{s s}$ and $B W$

\begin{tabular}{|c|c|c|c|c|c|c|c|c|}
\hline \multicolumn{2}{|c|}{ Column no. $(i) \rightarrow$} & 0 & 1 & 3 & \multirow{2}{*}{$\ldots$} & $C-4$ & $C-2$ & $C-1$ \\
\hline \multicolumn{2}{|l|}{$\downarrow$} & $\begin{array}{c}\{i<(C-I)\} \\
\&(i \% \\
3=0)\end{array}$ & $\begin{array}{c}\{i<(C-I)\} \\
\&(i \% 3=1)\end{array}$ & $\begin{array}{c}\{i<(C-I)\} \\
\&(i \% 3=2)\end{array}$ & & $\begin{array}{c}i>=(C-I) \\
\&(i \% 3=0)\end{array}$ & $\begin{array}{c}i>=(C-I) \\
\&(i \% 3=1)\end{array}$ & $\begin{array}{c}i>=(C-I) \\
\&(i \% 3=2)\end{array}$ \\
\hline 0 & $\begin{array}{l}\{j<(D-J)\} \\
\&(j \% 3=0)\end{array}$ & $\begin{array}{c}D^{*}(i+I)+ \\
\quad(j+J)\end{array}$ & $\begin{array}{c}D^{*}(i+I)+ \\
(j+J+2)\end{array}$ & $\begin{array}{c}D^{*}(i+I)+ \\
(j+J+1)\end{array}$ & \multirow{3}{*}{$\ldots$} & $\begin{array}{c}D^{*}\{i-(C-I)\}+ \\
(j+J)\end{array}$ & $\begin{array}{c}D^{*}\{i-(C-I)\}+ \\
\quad(j+J+2)\end{array}$ & $\begin{array}{c}D^{*}\{i-(C-I)\}+ \\
\quad(j+J+1)\end{array}$ \\
\hline 2 & $\begin{array}{l}\{j<(D-J)\} \\
\&(j \% 3=1)\end{array}$ & $\begin{array}{c}D^{*}(i+I)+ \\
\quad(j+J)\end{array}$ & $\begin{array}{c}D^{*}(i+I)+ \\
(j+J-1)\end{array}$ & $\begin{array}{c}D^{*}(i+I)+ \\
(j+J+1)\end{array}$ & & $\begin{array}{c}D^{*}\{i-(C-I)\}+ \\
(j+J)\end{array}$ & $\begin{array}{c}D^{*}\{i-(C-I)\}+ \\
(j+J-1)\end{array}$ & $\begin{array}{c}D^{*}\{i-(C-I)\}+ \\
(j+J+1)\end{array}$ \\
\hline 3 & $\begin{array}{l}\{j<(D-J)\} \\
\&(j \% 3=2)\end{array}$ & $\begin{array}{c}D^{*}(i+I)+ \\
\quad(j+J)\end{array}$ & $\begin{array}{c}D^{*}(i+I)+ \\
(j+J-1)\end{array}$ & $\begin{array}{c}D^{*}(i+I)+ \\
(j+J-2)\end{array}$ & & $\begin{array}{c}D^{*}\{i-(C-I)\}+ \\
(j+J)\end{array}$ & $\begin{array}{c}D^{*}\{i-(C-I)\}+ \\
(j+J-1)\end{array}$ & $\begin{array}{c}D^{*}\{i-(C-I)\}+ \\
\quad(j+J-2)\end{array}$ \\
\hline \multicolumn{2}{|r|}{$\ldots$} & $\begin{array}{c}i<(C-I-1) \\
\&(i \% \\
3=0)\end{array}$ & $\begin{array}{l}\{i<(C-I-1)\} \\
\&(i \% 3=1)\end{array}$ & $\begin{array}{l}\{i<(C-I-1)\} \\
\&(i \% 3=2)\end{array}$ & & $\begin{array}{l}i>=(C-I-1) \\
\&(i \% 3=0)\end{array}$ & $\begin{array}{l}i>=(C-I-1) \\
\&(i \% 3=1)\end{array}$ & $\begin{array}{l}i>=(C-I-1) \\
\&(i \% 3=2)\end{array}$ \\
\hline$D-4$ & $\begin{array}{l}\{j>=(D-J)\} \\
\&(j \% 3=0)\end{array}$ & $\begin{array}{l}D^{*}(i+I+1) \\
+\{j-(D-J)\}\end{array}$ & $\begin{array}{c}D^{*(i+I+1)+} \\
\{j-(D-J-2)\}\end{array}$ & $\begin{array}{c}D^{*}(i+I+1)+ \\
\{j-(D-J-1)\}\end{array}$ & & $\begin{array}{c}D^{*}\{i-(C-I-1)\}+ \\
\{j-(D-J)\}\end{array}$ & $\begin{array}{c}D^{*}(i-(C-I- \\
1))+(j-(D-J- \\
2))\end{array}$ & $\begin{array}{c}D^{*}(i-(C-I- \\
1))+(j-(D-J- \\
1))\end{array}$ \\
\hline$D-2$ & $\begin{array}{l}\{j>=(D-J)\} \\
\&(j \% 3=1)\end{array}$ & $\begin{array}{l}D^{*}(i+I+1) \\
+(j-(D-J))\end{array}$ & $\begin{array}{c}D^{*}(i+I+1)+ \\
(j-(D-J+1))\end{array}$ & $\begin{array}{c}D^{*}(i+I+1)+ \\
(j-(D-J-1))\end{array}$ & ;. & $\begin{array}{c}D *(i-(C-I-1))+ \\
\quad(j-(D-J))\end{array}$ & $\begin{array}{c}D^{*}(i-(C-I- \\
1))+(j-(D- \\
J+1))\end{array}$ & $\begin{array}{c}D^{*}(i-(C-I- \\
1))+(j-(D-J- \\
1)) \\
\end{array}$ \\
\hline$D-2$ & $\begin{array}{l}\{j>=(D-J)\} \\
\text { er ; of } 2=2\}\end{array}$ & $D^{*}(i+I+1)$ & $D^{*}(i+I+1)+$ & $D^{*}(i+I+1)+$ & & $\begin{array}{c}D^{*}(i-(C-I-1))+ \\
(i-(D-n)\end{array}$ & $D^{*}(i-(C-I-$ & $\begin{array}{l}D^{*}(i-(C-I- \\
1))+(j-(D-\end{array}$ \\
\hline
\end{tabular}




\begin{tabular}{|l|l|l|l|l|l|l|l|l|}
\hline$D-1$ & & & & & & & $J+1))$ & $J+2))$ \\
\hline
\end{tabular}

13 


\section{Table 3 (on next page)}

Values of $J_{\text {rot }}$ for all modulation schemes, spatial streams and BWs 
Table 3 Values of $J_{\text {rot }}$ for all modulation schemes, spatial streams and BWs

\begin{tabular}{lllllllll}
\hline \multirow{2}{*}{$\begin{array}{l}\text { Modulation Scheme } \\
\left(\mathrm{N}_{\mathrm{cbpsc}}\right)\end{array}$} & \multicolumn{2}{l}{$\mathrm{BW}=20 \mathrm{MHz}$} & \multicolumn{7}{c}{$\mathrm{BW}=40 \mathrm{MHz}$} \\
\cline { 2 - 9 } & $\mathrm{I}_{\mathrm{ss}}=1$ & $\mathrm{I}_{\mathrm{ss}}=2$ & $\mathrm{I}_{\mathrm{ss}}=3$ & $\mathrm{I}_{\mathrm{ss}}=4$ & $\mathrm{I}_{\mathrm{ss}}=1$ & $\mathrm{I}_{\mathrm{ss}}=2$ & $\mathrm{I}_{\mathrm{ss}}=3$ & $\mathrm{I}_{\mathrm{ss}}=4$ \\
\hline BPSK $\left(\mathrm{N}_{\mathrm{cbpsc}}=1\right)$ & 0 & 26 & 13 & 39 & 0 & 58 & 29 & 87 \\
QPSK $\left(\mathrm{N}_{\text {cbpsc }}=2\right)$ & 0 & 52 & 26 & 78 & 0 & 116 & 58 & 174 \\
16-QAM $\left(\mathrm{N}_{\mathrm{cbpsc}}=4\right)$ & 0 & 104 & 52 & 156 & 0 & 232 & 116 & 348 \\
64-QAM $\left(\mathrm{N}_{\text {cbpsc }}=6\right)$ & 0 & 156 & 78 & 234 & 0 & 348 & 174 & 522 \\
\hline
\end{tabular}

2

3 
Table 4(on next page)

Encoding of BW

Table 4(a) Encoding of BW Table 4(b) Encoding of $N_{c b p s c}$ 
1

2

3

4

5

6

7
Table 4(a) Encoding of $B W$

\begin{tabular}{cc}
\hline Bandwidth $(B W)$ & Encoded bit \\
\hline $20 \mathrm{MHz}$ & 0 \\
$40 \mathrm{MHz}$ & 1 \\
\hline & \\
Table 4(b) Encoding of $N_{c b p s c}$ \\
\hline $\begin{array}{c}\text { Modulation Scheme } \\
\left(N_{c b p s c}\right)\end{array}$ \\
Encoded bits \\
\hline BPSK $\left(N_{c b p s c}=1\right)$ & 00 \\
QPSK $\left(N_{c b p s c}=2\right)$ & 01 \\
16-QAM $\left(N_{c b p s c}=4\right)$ & 10 \\
64-QAM $\left(N_{c b p s c}=6\right)$ & 11 \\
\hline
\end{tabular}




\section{Table 5 (on next page)}

Definition of $I_{x}$ and $J_{y}$ for all streams and $B W$ 
Table 5 Definition of $I_{x}$ and $J_{y}$ for all streams and $B W$

2

\begin{tabular}{lll}
\hline Stream & $B W=20 M H z, C=13$ & $B W=40 M H z, C=18$ \\
\hline$I_{s s}$ & $I_{1}=0, J_{1}=0$ & $I_{1}=0, J_{1}=0$ \\
$I_{s s 2}$ & $I_{2}=6, J_{2}=N B P S C^{*} 2$ & $I_{2}=8, J_{2}=N B P S C^{*} 2$ \\
$I_{s s 3}$ & $I_{3}=9, J_{3}=N B P S C^{* 3}$ & $I_{3}=13, J_{3}=N B P S C$ \\
$I_{s s 4}$ & $I_{4}=3, J_{4}=N B P S C$ & $I_{4}=3, J_{4}=N B P S C^{*} 3$ \\
\hline
\end{tabular}


Table 6 (on next page)

Encryption of signals

Table 6(a) Encryption of signals II4 and JJ4 Table 6(b) Encryption of signals II6 and JJ6 
Table 6(a) Encryption of signals II4 and JJ4

2

\begin{tabular}{llll}
\hline Condition & $I I 4$ & Condition & JJ4 \\
\hline ICOUNT $<\left(C-I_{x}\right)$ and $i X M O D=0$ & 00 & $J C O U N T<\left(D-J_{y}\right)$ and $j X M O D=0$ & 00 \\
ICOUNT $<\left(C-I_{x}\right)$ and $i X M O D=1$ & 01 & JCOUNT $<\left(D-J_{y}\right)$ and $j X M O D=1$ & 01 \\
ICOUNT $\geq\left(C-I_{x}\right)$ and $i X M O D=0$ & 10 & JCOUNT $\geq\left(D-J_{y}\right)$ and $j X M O D=0$ & 10 \\
ICOUNT $\geq\left(C-I_{x}\right)$ and $i X M O D=1$ & 11 & JCOUNT $\geq\left(D-J_{y}\right)$ and $j X M O D=1$ & 11 \\
\hline
\end{tabular}

Table 6(b) Encryption of signals IIG and JJ6

\begin{tabular}{llll}
\hline Condition & $I I 6$ & Condition & JJ6 \\
\hline$I C O U N T<\left(C-I_{x}\right)$ and $i X M O D=0$ & 000 & $J C O U N T<\left(D-J_{y}\right)$ and $j X M O D=0$ & 000 \\
$I C O U N T<\left(C-I_{x}\right)$ and $i X M O D=1$ & 001 & $J C O U N T<\left(D-J_{y}\right)$ and $j X M O D=1$ & 001 \\
$I C O U N T<\left(C-I_{x}\right)$ and $i X M O D=2$ & 010 & $J C O U N T<\left(D-J_{y}\right)$ and $j X M O D=2$ & 010 \\
$I C O U N T \geq\left(C-I_{x}\right)$ and $i X M O D=0$ & 011 & $J C O U N T \geq\left(D-J_{y}\right)$ and $j X M O D=0$ & 011 \\
$I C O U N T \geq\left(C-I_{x}\right)$ and $i X M O D=1$ & 100 & JCOUNT $\geq\left(D-J_{y}\right)$ and $j X M O D=1$ & 100 \\
$I C O U N T \geq\left(C-I_{x}\right)$ and $i X M O D=2$ & 101 & JCOUNT $\geq\left(D-J_{y}\right)$ and $j X M O D=2$ & 101 \\
\hline
\end{tabular}




\section{Table 7 (on next page)}

Device Utilization Summary 
1

2
Table 7 Device Utilization Summary

\begin{tabular}{ccccc}
\hline \multirow{2}{*}{ FPGA Resources } & \multicolumn{2}{c}{ This work } & \multicolumn{2}{c}{ LUT Based technique } \\
\cline { 2 - 5 } & Utilization in Number & Utilization in $\%$ & Utilization in Number & Utilization in \% \\
\hline Number of Slices Registers & 30 out of 30064 & 0.10 & 35 out of 30064 & 0.12 \\
Number of Slices LUTs & 864 out of 15032 & 5.75 & 201 out of 15032 & 1.34 \\
Number of BRAMs & 4 out of 52 & 7.69 & 36 out of 52 & 69.23 \\
Number of DSP48A1s & 4 out of 38 & 10.53 & 0 out of 38 & $0 \%$ \\
Number of BUFG/BUFGCTRLs & 2 out of 16 & 12.50 & 2 out of 16 & 12.50 \\
\hline
\end{tabular}

3

4 


\section{Table 8 (on next page)}

Throughput comparison with IEEE 802.11n 
Table 8: Throughput comparison with IEEE 802.11n

\begin{tabular}{cccc}
\hline \multirow{2}{*}{$\begin{array}{c}\text { Maximum throughput } \\
\text { requirement of IEEE 802.11n }\end{array}$} & Modulation Scheme & Maximum throughput & \begin{tabular}{c} 
Improvement \\
over IEEE \\
\cline { 2 - 4 } $802.11 \mathrm{n}$
\end{tabular} \\
\hline \multirow{2}{*}{$600 \mathrm{Mbps}$} & BPSK & $834.8 \mathrm{Mbps}$ & 1.39 times \\
& QPSK & $1669.6 \mathrm{Mbps}$ & 2.78 times \\
& 16-QAM & $3339.2 \mathrm{Mbps}$ & 5.57 times \\
& 64-QAM & $5008.8 \mathrm{Mbps}$ & 8.35 times \\
\hline
\end{tabular}

2 


\section{Table 9 (on next page)}

Comparative study with similar works 
Table 9 Comparative study with similar works

2

\begin{tabular}{lccccc}
\hline \multicolumn{1}{c}{ FPGA Parameters } & This work & {$[15]$} & {$[7]$} & {$[16]$} & LUT Based \\
\hline $\begin{array}{l}\text { Maximum clock } \\
\text { frequency, } f\end{array}$ & $208.7 \mathrm{MHz}$ & $109.38 \mathrm{MHz}$ & $70.31 \mathrm{MHz}$ & $125 \mathrm{MHz}$ & $151.45 \mathrm{MHz}$ \\
Power consumption, $P$ & $28.62 \mathrm{~mW}$ & $111.24 \mathrm{~mW}$ & $48 \mathrm{~mW}$ & Not available & $28.62 \mathrm{~mW}$ \\
\hline
\end{tabular}

3 
Figure 1

Block diagram of steps involved in interleaving process for MIMO WLAN

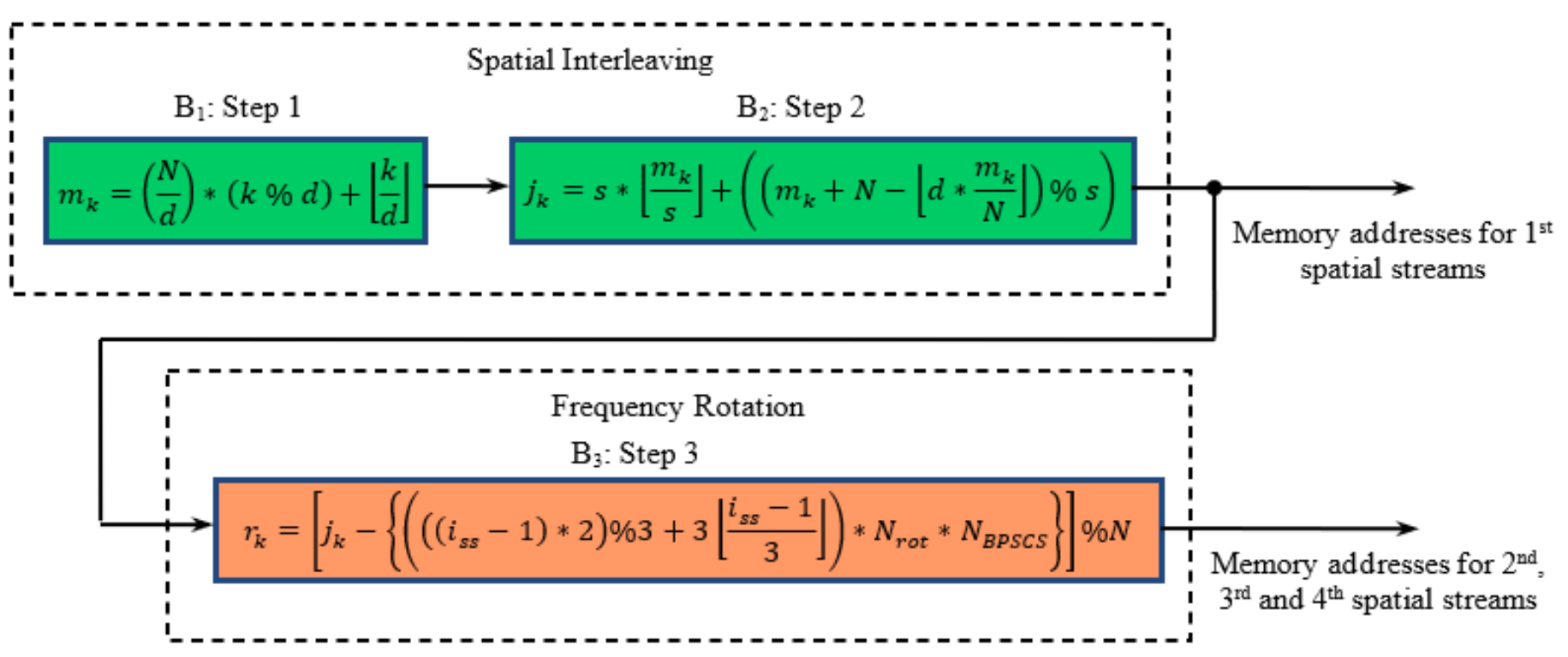


Figure 2

Top level view of complete interleaver

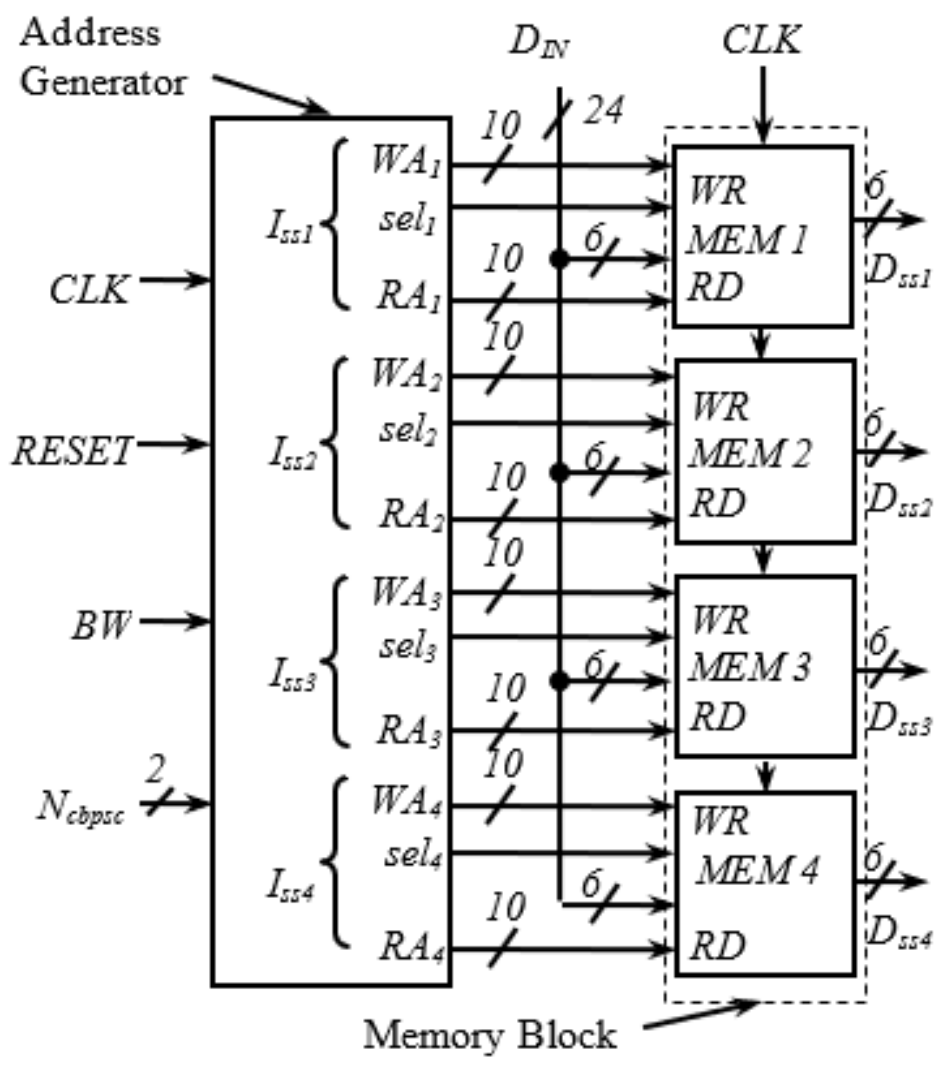


Figure 3

Internal structure of memory block

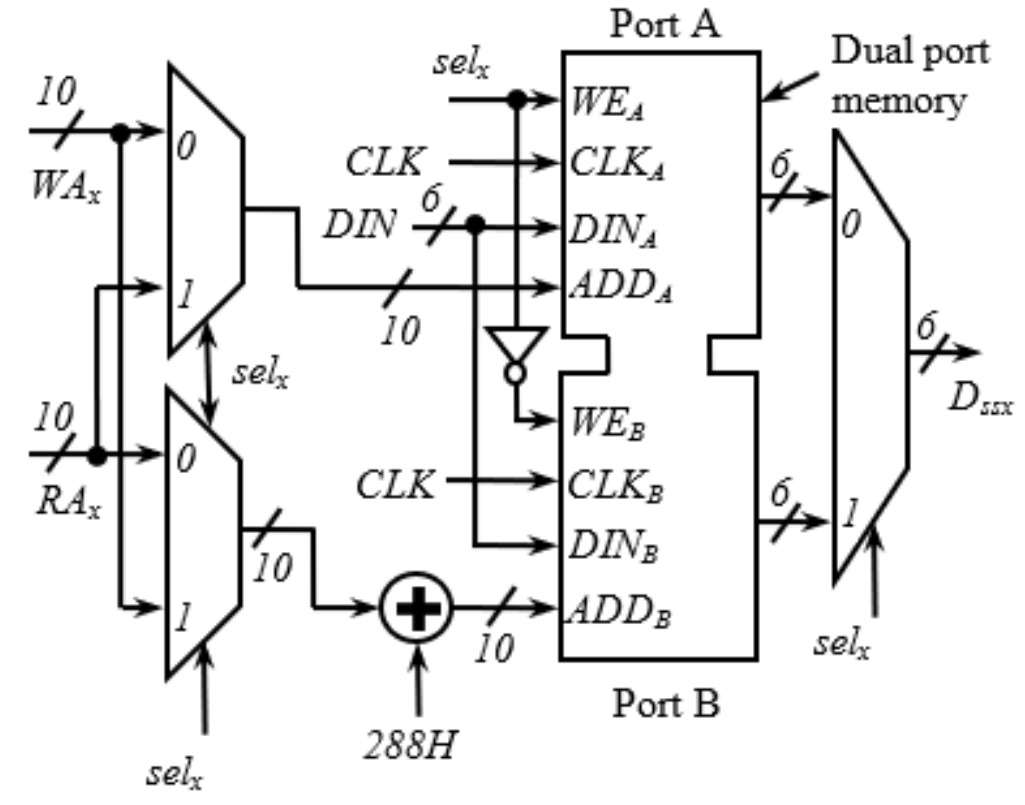


Figure 4

Multiplexing scheme showing write address generation for one spatial stream

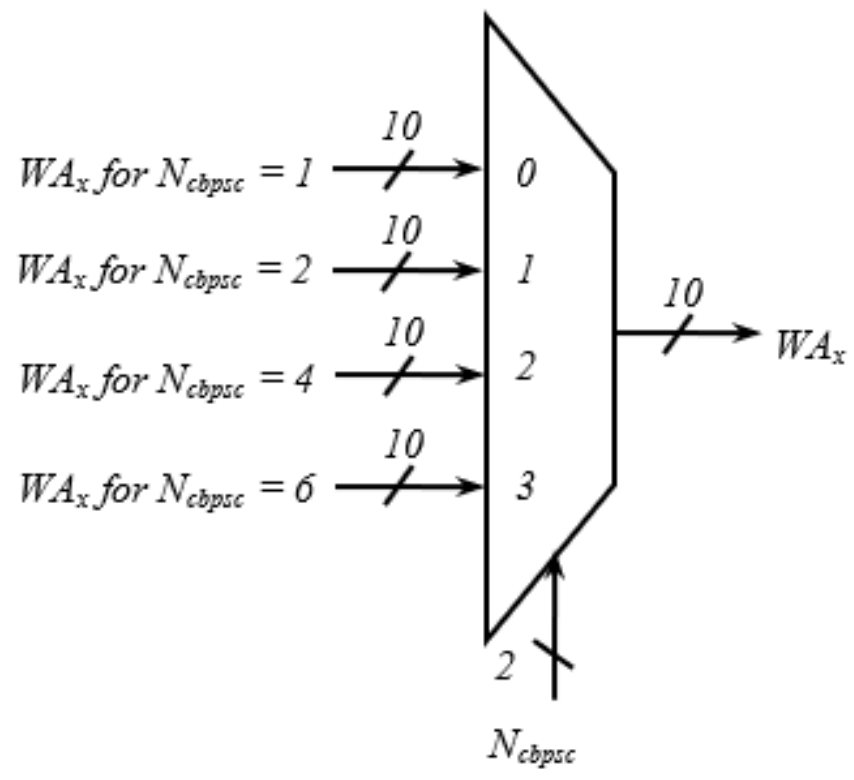


Figure 5

Scheme showing generation of (a) row count and (b) column count

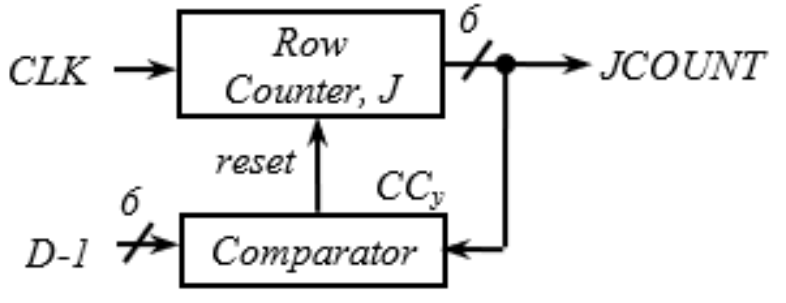

(a)

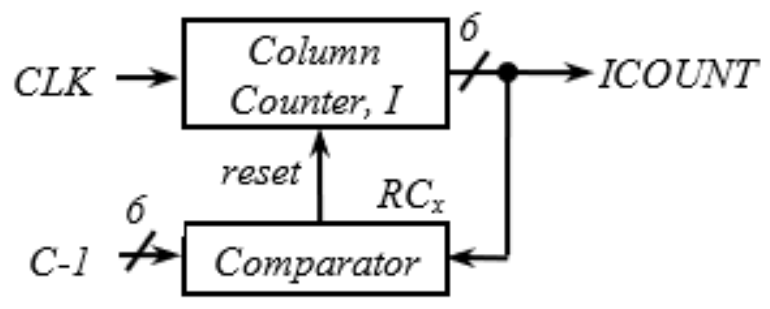

(b) 
Figure 6

Scheme for generation of number of rows $(D)$

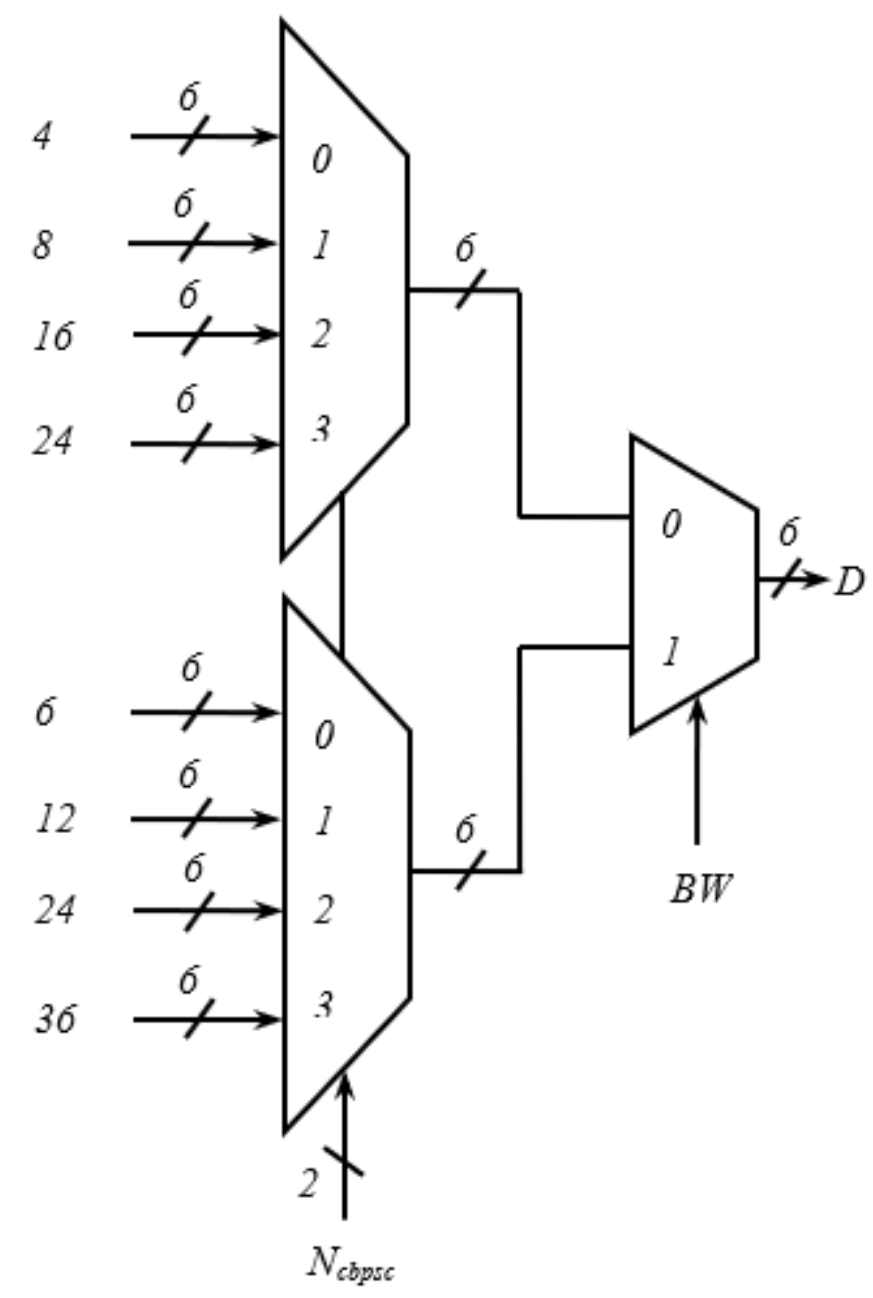


Figure 7

Arrangement showing generation of (a) ICOUNT $<\left(C-I_{x}\right)$ and ICOUNT $\geq\left(C-I_{x}\right)$ (b) JCOUNT $<\left(D-J_{y}\right)$ and JCOUNT $\geq\left(D-J_{y}\right)$

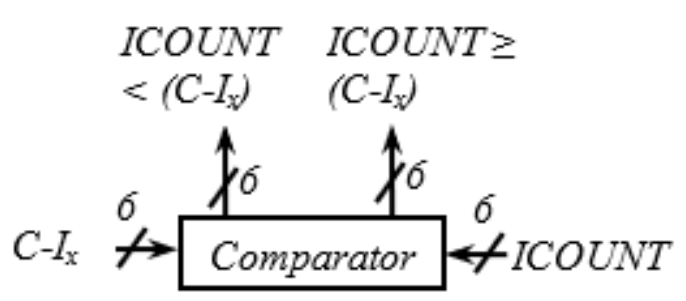

(a)

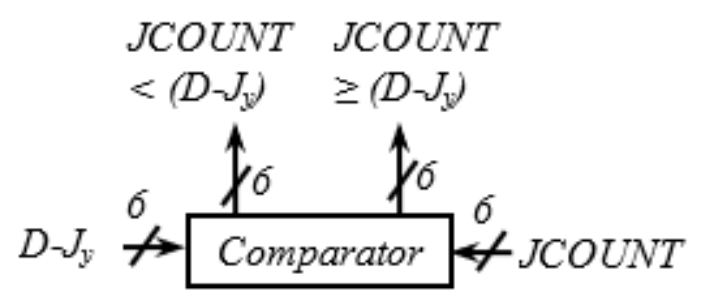

(b) 
Figure 8

Circuit for generation of read address $\left(R A_{x}\right)$

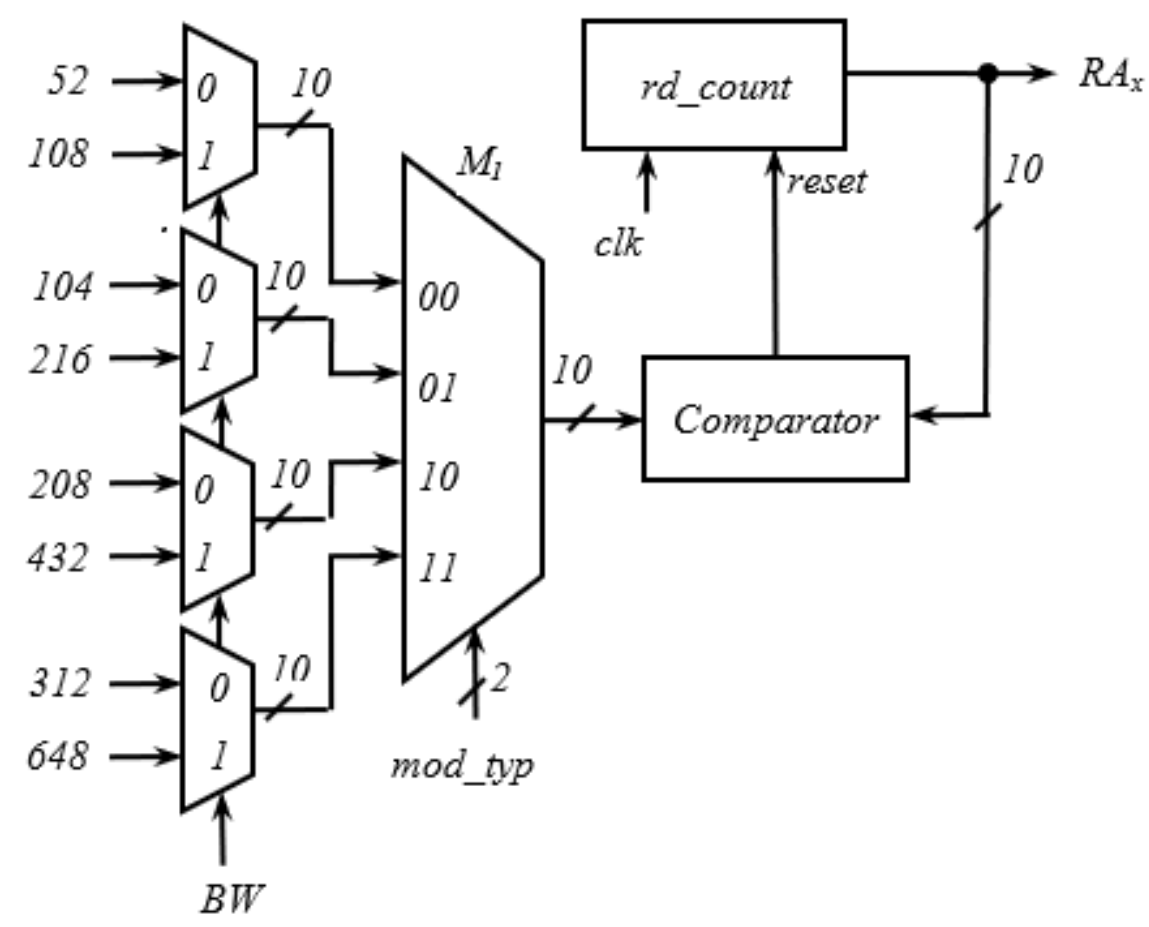


Figure 9

Circuit diagram for generation of interleaver write addresses with $N_{\text {cbpsc }}=1$ or 2

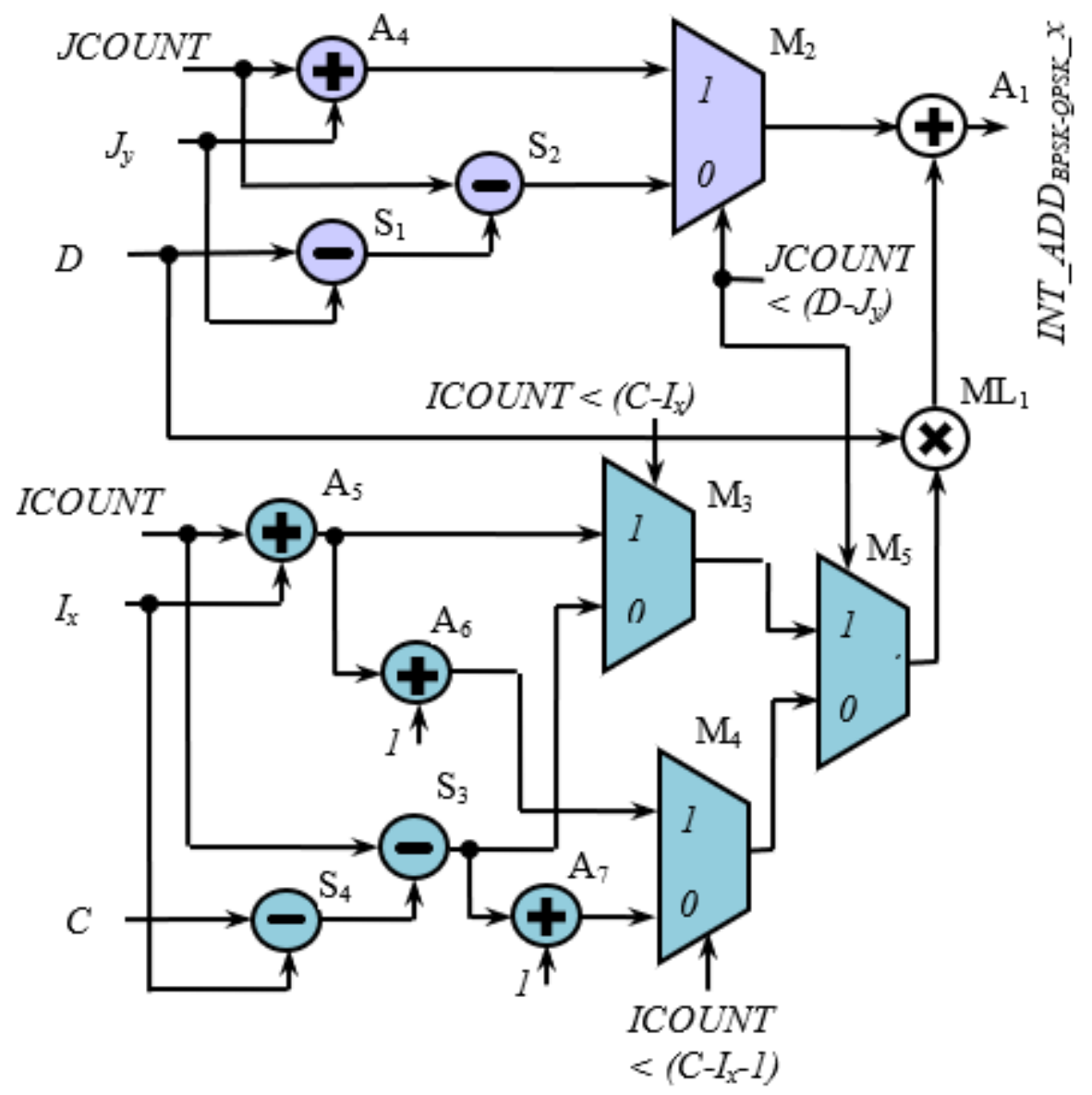


Figure 10

Circuit diagram for generation of interleaver write addresses with (a) $N_{c b p s c}=4$ and (b) $N_{\text {cbpsc }}=6$

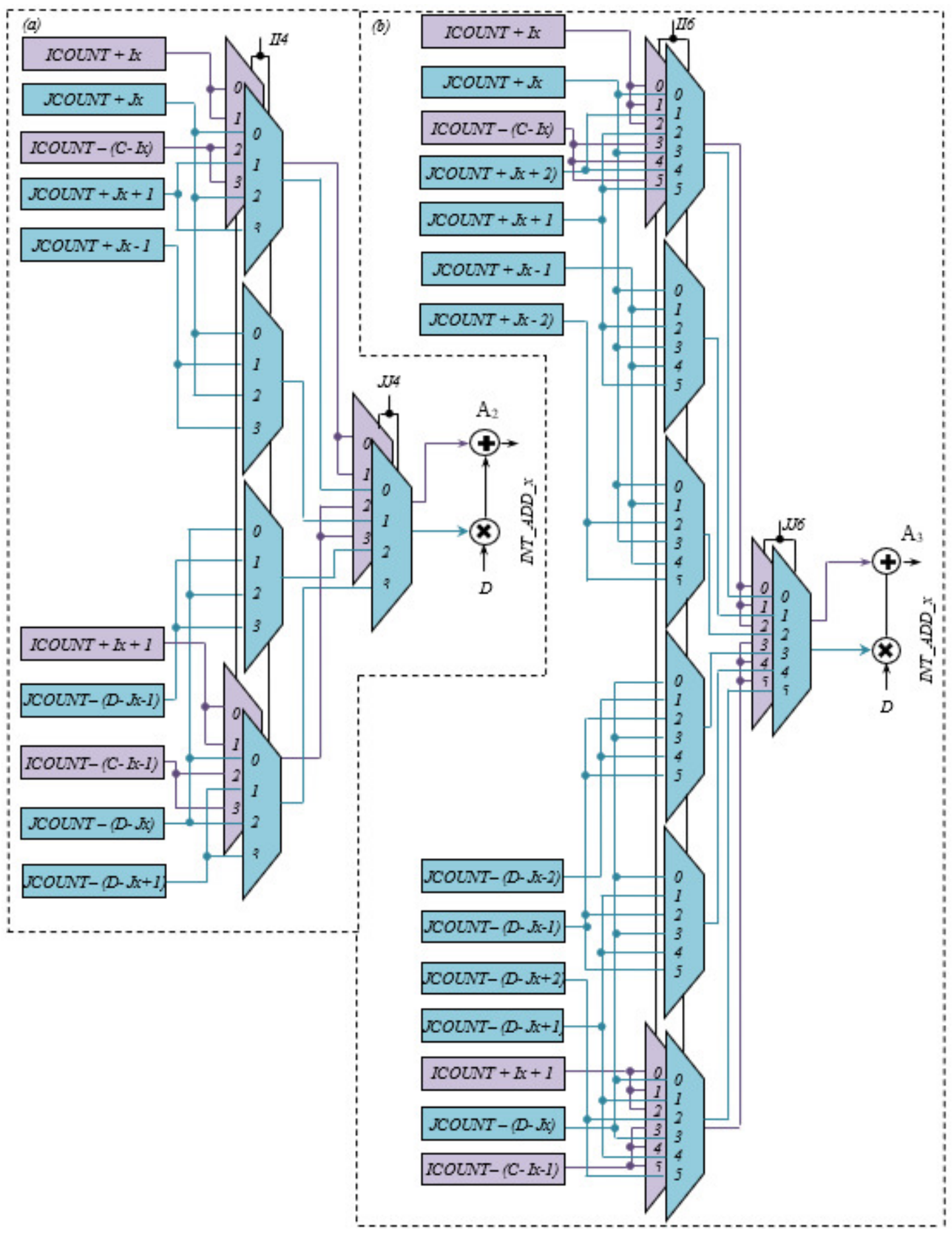




\section{Figure 11}

Write addresses $\left(W A_{x}\right)$ for (a) $B W=0(20 \mathrm{MHz}), N_{\text {bpscs }}=00$ (BPSK), (b) $B W=1(40 \mathrm{MHz})$, $N_{\text {bpscs }}=11$ (64-QAM)

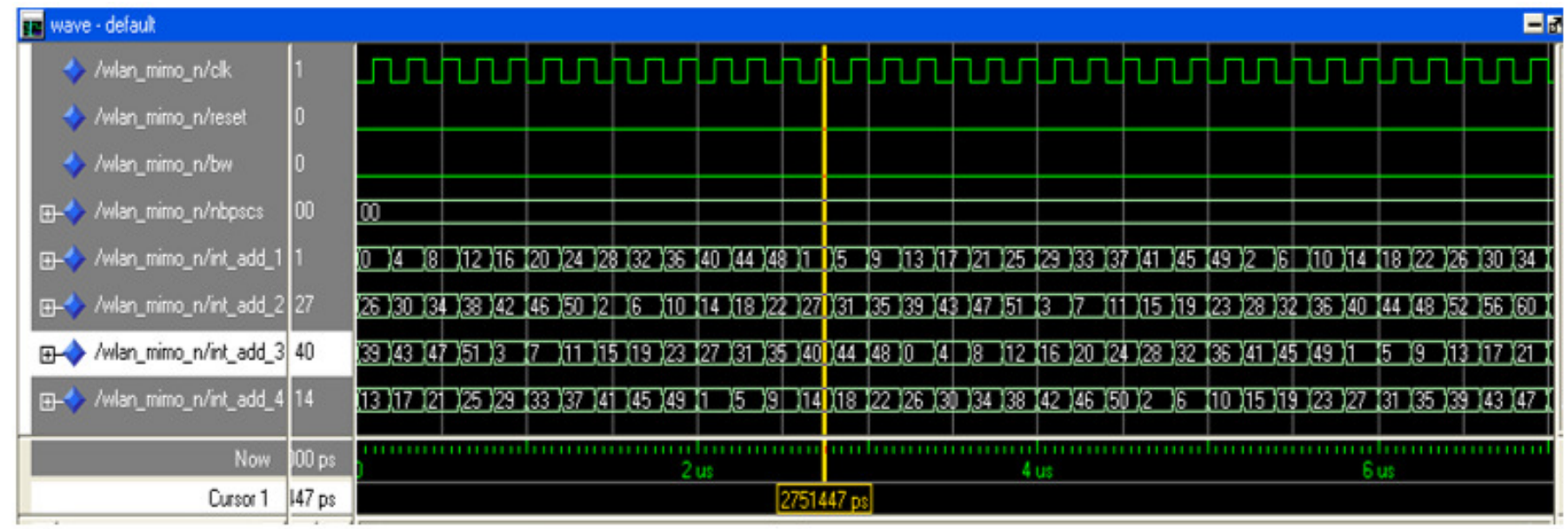

(a)
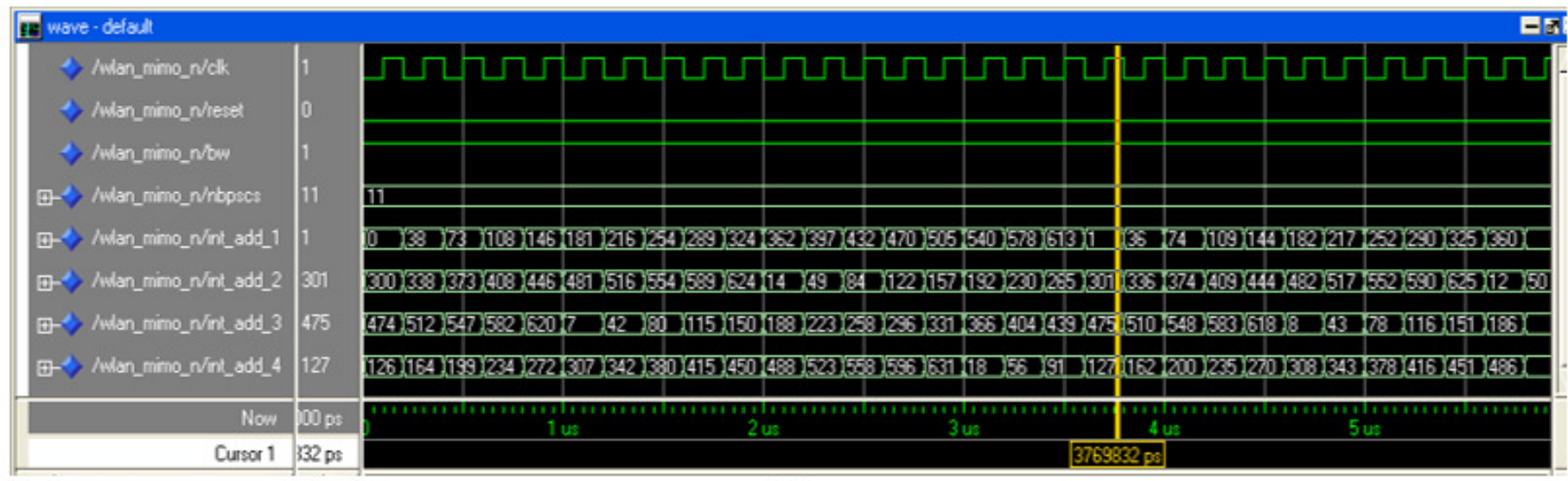

(b) 
Figure 12

Test arrangement of the address generator using VIO and ILA

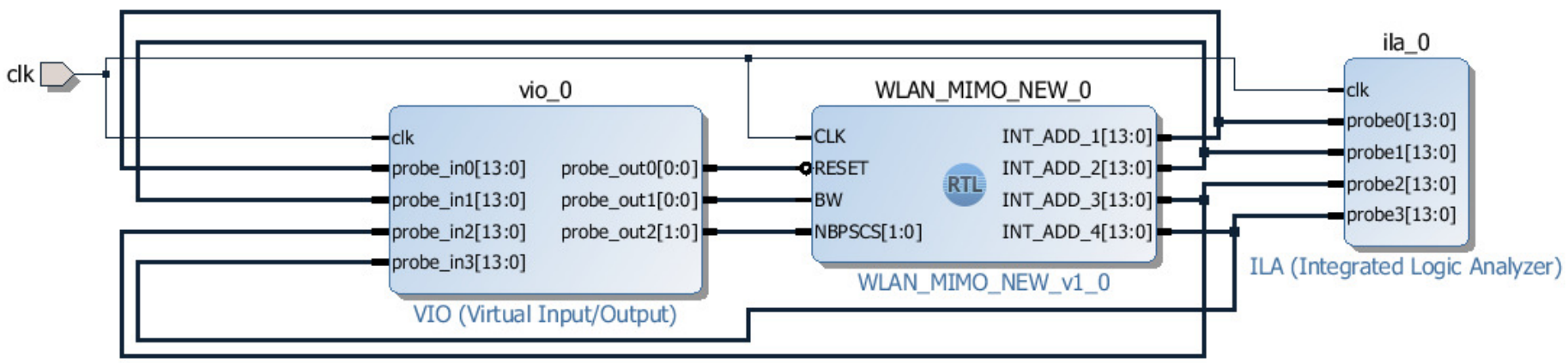


Figure 13

Performance comparison

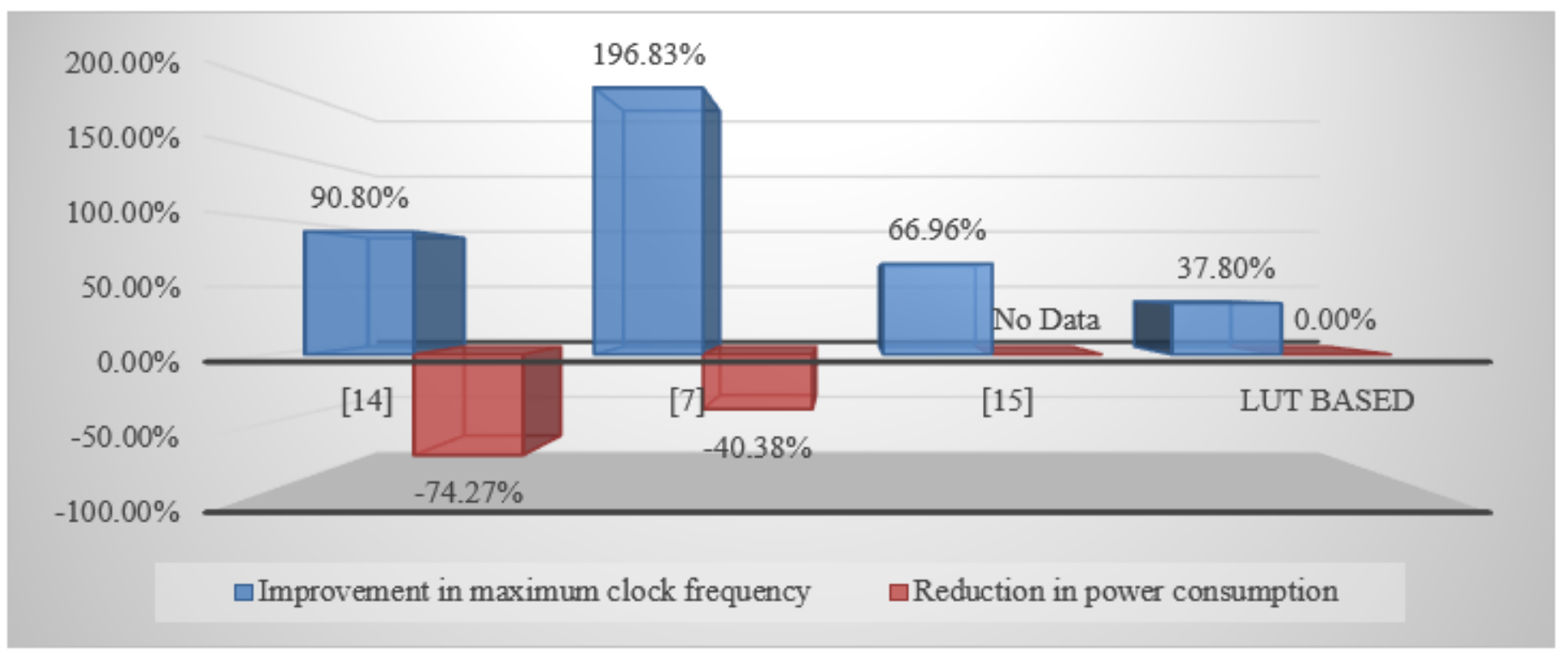

